UDC 615.454:661.185

DOI: $10.15587 / 2519-4852.2021 .249312$

\title{
INTERACTION OF SURFACTANTS WITH POLOXAMERS 338 AND ITS EFFECT ON SOME PROPERTIES OF CREAM BASE
}

\author{
Elena Bezuglaya, Nikolay Lyapunov, Oleksii Lysokobylka, Oleksii Liapunov, Vladimir Klochkov, \\ Ganna Grygorova, Anna Liapunova
}

The aim. Study of the interaction of surfactants with poloxamer 338 (P338) and the effect of P338 on the properties
of cream bases. Materials and methods. Solutions of the surfactants and P338 as well as cream bases were under study. The average hydrodynamic diameter $\left(D_{h}\right)$ and zeta potential ( $\zeta$-potential) were determined by the light scattering intensity and electrophoretic mobility of micelles. The electron paramagnetic resonance (EPR) spectra of spin probes in micelles, solvents and bases were obtained; the type of spectrum, isotropic constant $\left(A_{N}\right)$, rotational correlation times $(\tau)$ and anisotropy parameter $(\varepsilon)$ were determined. Liquids and cream bases were studied by capillary and rotational viscometry; the flow behaviour and yield stress $\left(\tau_{0}\right)$, dynamic and apparent viscosity $(\eta)$ as well as the hysteresis (thixotropic) area $\left(A_{H}\right)$ were determined. The microstructure of the bases was examined by optical microscopy. The strength of adhesion $\left(S_{m}\right)$ was assessed by the pull-off test, and the absorption of water was studied by dialysis.

Results. Under the impact of P338 the hydrodynamic diameters of micelles formed by cationic, anionic and nonionic surfactants decreased as well as the absolute values of their $\zeta$-potential became lower, but the microviscosity of the micelle nuclei increased. There was also a change in the structure of the aggregates of surfactant with fatty alcohols; EPR spectra, which were superpositions characteristic for the lateral phase separation, converted into triplets that indicated the uniform distribution of lipophilic probes in the surfactant phase. When the content of P338 increased to $17 \%$, the rheological parameters of the bases increased drastically, the flow behaviour and the microstructure changed. The bases had the consistency of cream within temperature range from $25{ }^{\circ} \mathrm{C}$ to $70{ }^{\circ} \mathrm{C}$ and completely restored their apparent viscosity, which had decreased under shear stress. P338 enhances the adhesive properties of the bases. Due to their microstructure, cream bases have a lower ability to absorb water compared to a solution and gel containing $17 \%$ and $20 \%$ P338, respectively.

Conclusions. The structure of surfactant micelles and aggregates of surfactants with fatty alcohols changed under impact of P338 due to the interaction of surfactants with P338. As a result of this interaction, at a sufficiently high concentration of $P 338$, the microstructure and flow behaviour of bases changed, their rheological parameters, which remain high at temperatures from $25^{\circ} \mathrm{C}$ to $70^{\circ} \mathrm{C}$, increased significantly, and water absorption parameters decreased. The bases with P338 were more adhesive

Keywords: poloxamer 338 (P338), surfactant, micelle, cream base, spin probe, rotational correlation time ( $\tau)$, rheological parameters

\author{
How to cite: \\ $10.15587 / 2519-4852.2021 .249312$ \\ (C) The Author(s) 2021 \\ This is an open access article under the Creative Commons CC BY license hydrate
}

Bezuglaya, E., Lyapunov, N., Lysokobylka, O., Liapunov, O., Klochkov, V., Grygorova, G., Liapunova, A. (2021). Interaction of surfactants with poloxamers 338 and its effect on some properties of cream base. ScienceRise: Pharmaceutical Science, 6 (34), 4-19. doi: http://doi.org/

\section{Introduction}

Poloxamers ( $\alpha$-hydro- $\omega$-hydroxypoly(oxyethylene) poly(oxypropylene) poly(oxyethylene) block copolymers) are block copolymers of ethylene oxide and propylene oxide represented by the following general formula [1, 2]:<smiles>CCC(C)(C)CCOCC(C)(CC)OC(C)CC(C)(O)CC</smiles>

Five different poloxamers, numbered 124, 188, 237, 338 and 407 are described in the monograph «Poloxamers» of the European Pharmacopoeia [1]. The first two digits multiplied by 100 give the approximate average molecular mass $\left(M_{r}\right)$ of the polyoxypropylene core of copolymer, and the last digit multiplied by 10 corresponds to the percentage by weight of the polyoxyethylene portion [2]. Many other types of poloxamers are commercially available, such as proxanol 268 , which is an ingredient of some semi-solid medicinal products registered in Ukraine and Russia [2-4].

Poloxamer 124 is a liquid, and poloxamers 188, 237, 338, 407 are white or almost white waxy powder, microbeads or flakes with a melting point of about $50{ }^{\circ} \mathrm{C}$. Poloxamer 188 is soluble in water; poloxamers 124, 237, 338,407 are very soluble in water and practically insoluble in light petroleum $[1,2]$. Poloxamers together with 
mixed solvents water - propylene glycol (PG) - macrogol 400 (M400) and $P G-M 400$ form different systems: clear liquids, clear gels, turbid unstable gels, turbid stable gels and solids, as shown by the example of proxanol 268 [5]. On the ternary phase diagrams the area of the region for each above-mentioned dispersed systems depended on the composition of the systems [5]. Therefore, poloxamers can be used in liquid, semi-solid and solid preparations.

Poloxamers are nonionic surface active substances, their polyoxyethylene chains is hydrophilic while the polyoxypropylene segment is hydrophobic. Due to their properties, they are used in pharmaceutical formulations [2]. The data on the functional purpose of poloxamers are summarizes in the literature [2]. They are used as emulsifiers (in particular, for fluorocarbons), solubilizer, gelling agents $(1,5-20 \%)$, stabilizing agents for dispersed systems (1-5\%), components of suppository bases $(46 \%$ or $90 \%)$, excipients for tablet technology (5$10 \%)$, in particular, for coating (10\%) [2]. Poloxamers may also be used as components of bases for ointments, creams and gels (5-20\%) [3-5]. Poloxamers have found use in preparations for the topical treatment of wounds due to their surface-active properties and ability to absorb a wound exudate $[3,4,6]$. Poloxamers may cause a formation of gels when heating their solutions (thermoreversible systems) [2]; studies regarding use of poloxamer 407 (thermogelling polymer) with cellulose derivatives for this purpose are described in the literature [7]. Study of emulgel systems containing poloxamer 407 and Carbopol $^{\circledR} 974 \mathrm{P}$ or polycarbophil was also performed [8].

On the other hand, it is surfactants that are the most commonly used stabilizing agents for heterogeneous dispersed systems with liquid dispersion medium [2]. For physical stabilization of $\mathrm{o} / \mathrm{w}$ emulsions a mixture of $\mathrm{o} / \mathrm{w}$ and w/o emulsifiers is used, which form mixed aggregates $[9,10]$. The structure of mixed aggregates of hydrophilic and lipophilic surfactants depends on their mass ratio, molecular structure and temperature. It was established by spin probes method that in mixed aggregates of emulsifiers, which differ significantly in the HLB values (for example, hydrophilic surfactants and higher aliphatic alcohols), in a certain temperature range there is a lateral phase separation when two-dimensionally solid-like and one-dimensionally liquid sites of higher fatty alcohols are formed near liquid sites of hydrophilic surfactants. In contrast to spherical micelles, in such aggregates, with increasing distance to the polar part, the packing density of alkyl chains of surfactants decreases, which indicates the nonspherical shape of these aggregates. Sufficient concentration of aggregates and their hydrophilicity provided by hydrophilic surfactants, nonspherical shape and solid-like sites cause the formation in the volume of dispersed systems (with liquid aqueous or water-glycol medium) coagulation structures. These structures are characterized by plastic or pseudoplastic flow behaviour, thixotropic properties and some apparent viscosity $[9,10]$. Due to those properties such dispersed systems could be used as vehicles (bases) for semi-solid preparations (SSPs).
Poloxamers as nonionic surfactants in aqueous solutions also form micelles, and at high concentrations they create lyotropic liquid crystals with different structure that leads to the formation of hydrogels [11]. Such ability as $s o l \rightarrow$ gel transition at increasing temperature, as well as capacity to solubilize of hydrophobic substances and incorporate nanoparticles in the micelles make it possible to use of poloxamers for long-term subcutaneous delivery systems and controlled release drugs [11-13]. Poloxamer-based hydrogels are attractive for ophthalmic, parenteral, transdermal, and vaginal administration. These hydrogels are liquid at room temperature but become more viscous at body temperature. In this case, gels remain at the application site for a longer time, and the release of active substance(s) is prolonged [13]. However, the prolonged release of active substances from such gels in situ is limited mainly by insufficient rheological and mechanical properties, which cause the rapid gel erosion and an elimination of barriers for the diffusion of active substances [14].

Therefore, it is necessary to find mechanisms for the enhancement of the rheological parameters of gels and reducing the release rate of active substance(s) in situ. Additional excipients [7, 12, 14] or chemically modified poloxamers $[14,15]$ can be used in order to achieve sustained drug release and prolong the presence of the gel at the site of its administration. The properties of the emulsions stabilized by poloxamer 188 may be affected by the localization of the molecules of active substance at the interface between the oil phase and dispersion media [16].

Poloxamers provide gels with a mucoadhesive effect and are promising in the composition of SSPs for application on mucous membranes [17, 18]. Gel consistency and mucoadhesive properties are important factors for the development of topical SSPs with poloxamers for administration in gynecology [19] and ophthalmology [20].

In aqueous solutions poloxamers could be as monomers, spherical micelles, lyotropic liquid crystals with various shapes: cubic, hexagonal and lamellar [11, 21, 22]. The self-assembly of poloxamer molecules in solutions depends on the mass ratio between the hydrophobic polyoxypropylene block and hydrophilic polyoxyethylene blocks, the molecular weight of the poloxamer, its content and solution temperature $[11,21]$. The effect of non-aqueous solvents (glycerin, PG, ethanol, glycerol triacetate) and surfactants (nonionic, anionic and cationic) on the formation of various lyotropic liquid crystalline microstructures with poloxamer 407 was also studied; the small angle X-ray scattering method and ternary phase diagrams were used for the research [21]. The effect of those cosolvents and surfactants on the phase behaviour of poloxamer 407 has been shown [21]. The influence of polysorbates on the properties of poloxamer 407 micelles was studied; it has been shown that polysorbate molecules are incorporated into poloxamer micelles and change the structure of micelle nuclei [23].

The results of studies presented in the scientific literature mainly concern the effect of surfactants and other excipients on the properties of poloxamer solutions, but not the effect of poloxamers on surfactant associates $[2,9,10]$. The use of surfactants in combination with 
poloxamers in semi-solid preparations intended for different routes of administration is not substantiated. Such studies should be aimed primarily at developing hydrophilic bases with sufficient rheological parameters to prevent rapid erosion and mechanical destruction of SSP in situ. These bases must also possess bioadhesive properties and be able to prolong release of active substances. Accordingly, the aim of the study was established.

The aim was to study of the interaction of surfactants with poloxamer 338 (P338) and the effect of P338 on the properties of cream bases.

\section{Planning (methodology) of the research}

It was planned to use cationic (cetylpyridinium chloride), anionic (sodium lauryl sulfate), nonionic (macrogol 20 cetostearyl ether) surfactants and P338. The objects of the research were:

1) surfactant solutions, P338 solutions or solutions of surfactants with P338 in water and in the mixed solvents $P G-M 400$ - water;

2) relevant disperse systems containing a mixture of surfactant with cetostearyl alcohol or a mixture of surfactant with P338 and cetostearyl alcohol;

3) corresponding to disperse systems emulsions $\mathrm{o} / \mathrm{w}$. It was necessary to vary the composition of solutions and disperse systems, in particular, the content of P338.

Depending on the P338 content, it was necessary to determine the hydrodynamic diameter of surfactant micelles, their $\zeta$-potential as well as rotational correlation parameters of the fatty acid-based spin probe. The results of these studies provided the information about changes in the structure of surfactant micelles under the influence of P338, which could demonstrate their interaction.

It was planned to study the rheological properties of disperse systems with liquid dispersion medium depending on the P338 concentration by the rotational viscometry. In order to study the mechanism of influence of P338 on the rheological properties of disperse systems, it was necessary to determine the dynamic viscosity of liquids, which were the dispersion medium, as well as the rotational correlation parameters of the spin probes:

1) hydrophilic probe TEMPON localized in dispersion medium;

2) fatty acid-based spin probes localized in the micelles and in the aggregates of emulsifiers.

In the case of disperse systems with high rheological parameters, it was necessary to study properties which are important for SSP: apparent viscosity depending on temperature and long-continued shear stress, microstructure, adhesion (compared to P338 solutions and disperse system without P338) as well as the kinetics of water absorption by dialysis (compared to solution and gel containing P338).

Comparative studies of the release of PG, M400 and active substances from dispersed systems, solutions and gels by dialysis are also essential. But the results of such studies will be the subject of other publications. To complete the complex studies, it was necessary to compare the rheological behaviour of disperse systems without oil phase and emulsions $\mathrm{o} / \mathrm{w}$, containing the emulsifier o/w, cetostearyl alcohol and P338.
All these comprehensive studies were essential to find out mechanisms of formation of hydrophilic bases for SSP which are characterized by high rheological parameters, significant bioadhesive properties, moderate ability to dehydrate biological objects and prolonged release of active substances and hydrophilic solvents.

\section{Materials and methods}

Cetylpyridinium Chloride (CPC) («Merck»), Sodium Laurilsulfate (Kolliphor ${ }^{\circledR}$ SLS) (SLS) («BASF»), Macrogol Cetostearyl Ether (Kolliphor ${ }^{\circledR}$ CS 20) (MCE) («BASF»), Poloxamer 338 (Kolliphor ${ }^{\circledR}$ P 338) (P338) («BASF»), Propylene Glycol (Kollisolv ${ }^{\circledR}$ PG) (PG) («BASF»), Macrogol 400 (Kollisolv ${ }^{\circledR}$ PEG 400) (M400) («BASF»), Cetostearyl Alcohol (Kolliwax ${ }^{\circledR}$ CSA 50) (CSA) («BASF»), Paraffin Liquid, Paraffin White Soft and purified water (hereinafter the water) were used in the experiments [1].

These substances met the requirements of the monographs of the European Pharmacopoeia [1]. In addition, a complex emulsifier Kolliphor ${ }^{\circledR}$ CSL («BASF»), which is a mixture of SLS and sodium cetostearyl sulfate with CSA, was used.

Dynamic light scattering (DLS) instrument «ZetaPALS Zeta Potential Analyzer» («Brookhaven Instruments Corporation»; software 9kpsdw32 - 5.33 version) was used to measure the hydrodynamic diameter $\left(D_{h}\right)$ and the polydispersity index (PDI). The fluctuations in the light intensity due to Brownian motion of the micelle in the solution were used to determine the intensity weighted average translational diffusion coefficients. The average hydrodynamic diameter was estimated from the diffusion coefficient using the Stokes-Einstein equation:

$$
D_{h}=k_{B} T / 3 \pi \eta D
$$

where $D$ is the diffusion coefficient, $k_{B}$ is the Boltzmann constant, $T$ is the absolute temperature, and $\eta$ is the viscosity of the medium.

For each experiment, the solution was pipetted into a disposable plastic vial before measuring the intensity of the auto-correlation function at scattering angle of $90^{\circ}$. All the measurements were conducted at temperature of $25^{\circ} \mathrm{C}$, which was automatically controlled by the instrument. DLS measurements were performed by ten consecutive runs on individual sample, $30 \mathrm{~s}$ each.

The $\zeta$-potential $(\zeta)$ was obtained through measurement of the electrophoretic mobility of the micelle using same equipment «ZetaPALS Zeta Potential Analyzer» (software palsw32-5.75 version). All investigations were performed at $25^{\circ} \mathrm{C}$. The zeta potential was automatically calculated from the electrophoretic mobility based on Smoluchowski's formula:

$$
\zeta=4 \pi \times \eta \times(v / E) / \varepsilon
$$

where $\eta$ is the dynamic viscosity of medium, $(v / E)$ is the electrophoretic mobility, $\varepsilon$ is the dielectric constant.

The kinematic viscosity (v) of liquids was determined by capillary viscometer method and their densi- 
ty $(\rho)$ was measured using oscillation-type density meter DMA 500 («Anton Paar GmbH», Austria; software version V1.003). The dynamic viscosity $(\eta)$ of liquids was calculated using the equation:

$$
\eta=v \cdot \rho
$$

Rheograms (plots of the shear stress $(\tau)$ vs the shear rate $\left(D_{r}\right)$ ) were obtained at certain temperature by rotational viscometry [1] using a rotating viscometer «Rheolab QC» with coaxial cylinders CC-27 (for creams and gels) and DG42 (for liquids) («Anton Paar GmbH»; software RHEOPLUS, 2.66 version). Rheograms were used to characterize the flow behaviour as well as to determine the apparent viscosity, hysteresis (thixotropic) area $\left(A_{H}\right)$, yield stress $\left(\tau_{0}\right)$ of bases or the dynamic viscosity of Newtonian liquids $(\eta)$.

The EPR spectroscopy was used for the research [24]. The following spin probes were used for the experiments:

- (1): 1-piperidinyloxy,4-(hexadecyldimethylammonio)-2,2,6,6-tetramethyl-, iodide $\left(M_{r}\right.$ 551,65; CAS: [114199-16-5]);

- (2): 4-palmitamido-2,2,6,6-tetramethylpiperidine-1-oxyl ( $M_{r}$ 409,67; CAS: [22977-65-7]);

-(3): 5-DOXYL Stearic acid $\left(M_{r} 384,57\right.$; CAS: [29545-48-0]);

- (4): 16-DOXYL Stearic acid $\left(M_{r} 384,57\right.$; CAS: [53034-38-1]).

- (5): TEMPON (4-oxo-TEMPO) $\left(\mathrm{C}_{9} \mathrm{H}_{16} \mathrm{NO}_{2}\right.$; $M_{r}$ 170,23; CAS: [2896-70-0]).

Probe 1 simulated the emulsifier o/w, and probes 2 , 3 and 4 served as models of the emulsifiers w/o. The free radical in probes 2, 3 and 4 are located near different carbon atoms of the alkyl chains. Probe 5 is hydrophilic, water-soluble substance, and its EPR spectra provide information about the dispersion medium.

The probes were added into the disperse systems at the concentration of $10^{-4} \mathrm{~mol} / \mathrm{l}$. EPR spectra were obtained using an EPR Spectrometer CMS8400 («Adani»).

The type of EPR spectrum (triplet, anisotropic spectrum, singlet, superposition spectrum) was determined. Using the EPR spectra, which were triplets, the hyperfine splitting constant $\left(A_{N}\right)$, which characterizes the micropolarity of the environment in the vicinity of radical, the peak heights as well as the width of the low-field component $\left(\Delta H_{+1}\right)$ and central component $\left(\Delta H_{0}\right)$ were determined.

Rotational correlation times of spin probes $\left(\tau_{+1}\right.$, $\left.\tau_{-1}, \tau_{ \pm 1}\right)$ and anisotropy parameter $(\varepsilon)$ were calculated by the following equations $[24,25]$ :

$$
\begin{aligned}
& \tau_{+1}=\left(\sqrt{\frac{h_{0}}{h_{+1}}}-1\right) \cdot \Delta H_{0} / 2 \cdot 10^{8} \\
& \tau_{-1}=\left(\sqrt{\frac{h_{0}}{h_{-1}}}-1\right) \cdot \Delta H_{0} / 3.6 \cdot 10^{9} \\
& \tau_{ \pm 1}=\left(\sqrt{\frac{h_{+1}}{h_{-1}}}-1\right) \cdot \Delta H_{+1} \cdot 6.65 \cdot 10^{-10}
\end{aligned}
$$

$$
\varepsilon=\frac{\sqrt{h_{0} / h_{+1}}-1}{\sqrt{h_{0} / h_{-1}}-1},
$$

where $h_{+1}, h_{0}$ and $h_{-1}$ are the the peak-to-peak heights of the low-field, central and high-field components of EPR spectrum; $\Delta H_{+1}$ and $\Delta H_{0}$ are the width of low-field and central components, respectively.

The reorientation of nonspherical molecules dissolved in a liquid is characterized by two different correlation times: $\tau_{-1}$, which is associated with fluctuations in directions perpendicular to the long axis of the probe, and $\tau_{+1}$, which is associated with its rotation around the long axis [25]; both of these parameters are taken into account in the case of $\tau_{ \pm 1}$ [24]. The rotational correlation time of probes $(\tau)$ is directly proportional to the effective radius of the molecule $(R)$ and to the microviscosity of its local surrounding $(\eta)$ and inversely proportional to the absolute temperature $(T)[25]$ :

$$
\tau=\left(4 \pi R^{3} \eta\right) / 3 k T
$$

The strength of adhesion was determined by pulloff test [26]. The adhesion strength $\left(S_{m}\right)$ was estimated as separation force $\left(F_{m}\right)$ per unit of contact area $\left(A_{0}\right)$ and calculated by the formula:

$$
S_{m}=F_{m}: A_{0}
$$

The absorption of water by the bases was studied by dialysis using cellulose membranes (GOST 7730-89), which were pre-soaked in water $P$ for 24 hours. A vertical diffusion chambers were used. The chamber with tested sample (3.0 g) was weighed at regular intervals and the mass of absorbed water was calculated by changing the mass of the contents of the chamber [6].

Unless otherwise stated, the experiments were performed at $25^{\circ} \mathrm{C}$. A circulating thermostat Julabo F12-ED («Julabo Labortechnik GmbH», Germany) was used to maintain a necessary temperature.

The microstructure of the systems was determined by optical microscopy $[1,27]$ using the microscope with micrometer «Krüss MBL-2100» («A. Krüss Optronic», Germany).

P338 was dissolved in water at $6-8^{\circ} \mathrm{C}$ with degassing of the solution. The surfactants were dissolved in water or in mixed solvent water $-P G-M 400$ at 50$60{ }^{\circ} \mathrm{C}$, and then cooled to $20-25^{\circ} \mathrm{C}$. If lipophilic component (CSA and liquid paraffin) was added into a disperse system, the mixture of water, surfactant and lipophilic component(s) was heated to $70-75^{\circ} \mathrm{C}$, stirred until the surfactant was dissolved and CSA was melted; then emulsifying was performed with degassing (phase A). A mixture of PG, M400 and P338 was melted at $65-70{ }^{\circ} \mathrm{C}$ while stirring and degassing (phase B). Phases A and B were mixed, homogenized and degassed at about 65 $70{ }^{\circ} \mathrm{C}$ and then cooled with stirring to ambient temperature. The compositions of the samples under study are given in the text of the article. 


\section{Research results}

Study of the effect of P338 on the structure of micelles and aggregates of surfactants with CSA

The results of determination of the average hydrodynamic diameter $\left(D_{h}\right)$, polydispersity (PDI) and $\zeta$-potential of micelles in $1 \%$ aqueous solutions of CPC, SLS and MCE, which contain P338 at different concentrations, are presented in Table 1 . The representative histograms characterizing the distribution of micelles by their hydrodynamic diameter in aqueous solutions can be seen in Fig. 1.

As can be seen from Table 1 , the $D_{h}$ value for the micelles of non-ionic surfactant MCE was approximately 16.7 times smaller than for CPC micelles and 18.5 times smaller than for SLS micelles.

P338 in aqueous solutions also forms micelles. At $25^{\circ} \mathrm{C}$ the average hydrodynamic diameter of P338 micelles in $5 \%$ aqueous solution was $514.24 \pm 18.60 \mathrm{~nm}$ (with PDI=0.417), and the $\zeta$-potential was $-22.09 \pm 1.70 \mathrm{mV}$.

When P338 was added to aqueous solutions of surfactants, a tendency to decrease the hydrodynamic diameter of micelles and increase their polydispersity index was observed (Table 1). For example, as the P338 content increased to $5 \% D_{h}$ value for the micelles of CPC, SLS, and MCE decreased 1.5, 1.5, and 3 times, respectively.

CPC micelles and SLS micelles in aqueous solutions were characterized by positive and negative values of $\zeta$-potential, respectively (Table 1 ). The micelles of MCE and P338 also had negative $\zeta$-potential, but they absolute values were 2.7 and 3.0 times less than for the SLS micelles, respectively. P338 affected $\zeta$-potential of micelles (Table 1). With the presence of P338 and increasing its concentration to $5 \% \zeta$-potentials of micelles changed: in the case of CPC from $70.74 \mathrm{mV}$ to $10.62 \mathrm{mV}$, in the case of SLS from $-65.80 \mathrm{mV}$ to $-15.09 \mathrm{mV}$, in the case of MCE from $-24.57 \mathrm{mV}$ to $-10.91 \mathrm{mV}$.

According to Table 1, it can be assumed that $D_{h}$ of micelles increased, and their surface was neutralized due to the interaction of P338 molecules with surfactant micelles. According to the literature [11, 21, 23], they might form mixed micelles. But the hydrodynamic diameter of such mixed micelles was due to the colloidal surfactant, not the block copolymer. This is most evident in the example of aqueous solutions containing nonionic surfactant MCE and P338. As can be seen in Table 1 and Fig. 1, in the mixed solutions containing $1 \% \mathrm{MCE}$ and P338 there were no associates with a large hydrodynamic diameter of about $500 \mathrm{~nm}$, characteristic for P338 micelles. That is, surfactants prevented the formation of P338 micelles. In mixed micelles, the interaction between ions or molecules of surfactant and P338 molecules may occur due to ion-dipole, hydrophobic and steric interactions. Probably the most significant is the ion-dipole interaction of P338 with CPC.

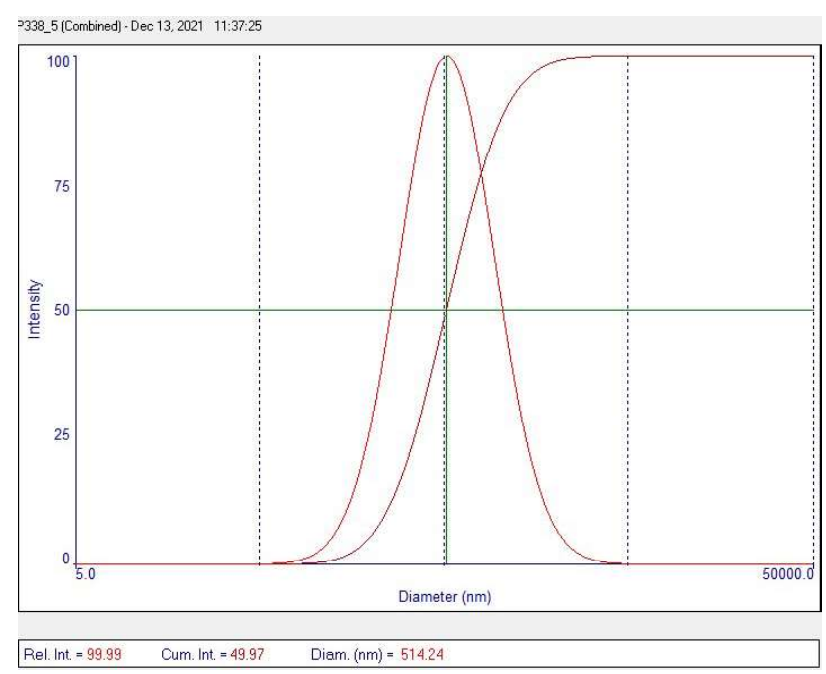

$a$

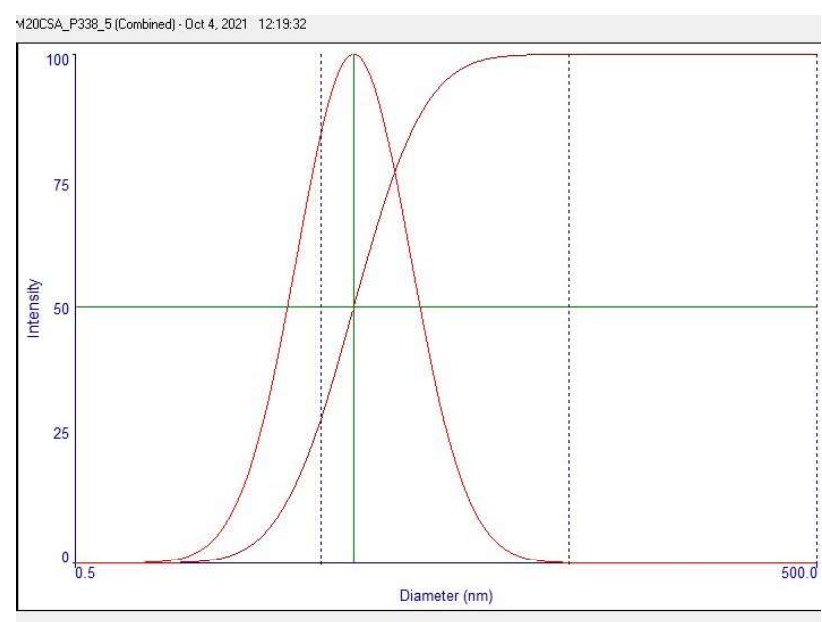

Rel. Int $=100.00 \quad$ Cum. Int $=50.43 \quad$ Diam. $(\mathrm{nm})=6.74$

$b$

Fig. 1. Lognormal distribution of micelles by hydrodynamic diameter $\left(D_{h}\right)$ in aqueous solutions containing $5 \%$ P338 (a) or $1 \%$ MCE with $5 \%$ P338 (b)

Table 1 $D_{h}$, PDI and $\zeta$-potential of micelles in $1 \%$ aqueous solutions of CPC, SLS and MCE with different content of P338

\begin{tabular}{|c|c|c|c|c|c|c|}
\hline \multirow{2}{*}{$\begin{array}{c}\text { Param- } \\
\text { eter }\end{array}$} & $0 \%$ & $1.0 \%$ & $2.0 \%$ & $3.0 \%$ & $4.0 \%$ & $5.0 \%$ \\
\hline \multicolumn{7}{|c|}{ Cetylpyridinium chloride (CPC) } \\
\hline$D_{h}, \mathrm{~nm}$ & $332.77 \pm 7.5$ & $282.67 \pm 11.51$ & $266.25 \pm 28.91$ & $253.40 \pm 23.21$ & $243.50 \pm 20.10$ & $221.47 \pm 21.8$ \\
\hline PDI & 0.321 & 0.358 & 0.379 & 0.409 & 0.396 & 0.424 \\
\hline$\zeta, \mathrm{mV}$ & $70.74 \pm 0.36$ & $39.80 \pm 4.11$ & $28.91 \pm 7.61$ & $15.48 \pm 7.24$ & $11.93 \pm 1.75$ & $10.62 \pm 3.10$ \\
\hline \multicolumn{7}{|c|}{ Sodium laurilsulfate (SLS) } \\
\hline$D_{h}, \mathrm{~nm}$ & $367.79 \pm 23.87$ & $331.38 \pm 17.78$ & $310.21 \pm 7.9$ & $300.34 \pm 30.51$ & $271.14 \pm 13.59$ & $251.30 \pm 23.96$ \\
\hline PDI & 0.331 & 0.326 & 0.290 & 0.368 & 0.401 & 0.401 \\
\hline$\zeta, \mathrm{mV}$ & $-65.80 \pm 3.70$ & $-29.26 \pm 2.04$ & $-24.81 \pm 3.80$ & $-21.70 \pm 2.73$ & $-16.33 \pm 2.65$ & $-15.09 \pm 7.71$ \\
\hline \multicolumn{7}{|c|}{ Macrogol 20 cetostearyl ether (MCE) } \\
\hline$D_{h}, \mathrm{~nm}$ & $19.93 \pm 0.10$ & $12.97 \pm 0.17$ & $9.76 \pm 0.06$ & $9.40 \pm 0.14$ & $7.58 \pm 0.11$ & $6.74 \pm 0.15$ \\
\hline PDI & 0.195 & 0.221 & 0.261 & 0.284 & 0.300 & 0.320 \\
\hline$\zeta, \mathrm{mV}$ & $-24.57 \pm 1.64$ & $-21.18 \pm 2.99$ & $-19.61 \pm 1.83$ & $-14.77 \pm 5.10$ & $-13.65 \pm 4.65$ & $10.91 \pm 10.16$ \\
\hline
\end{tabular}


The effect of P338 on the micelle structure was studied by spin probe method. The EPR spectra of spin probe 2 in different objects are illustrated in Fig. 2; some parameters of these EPR spectra are provided Table 2.

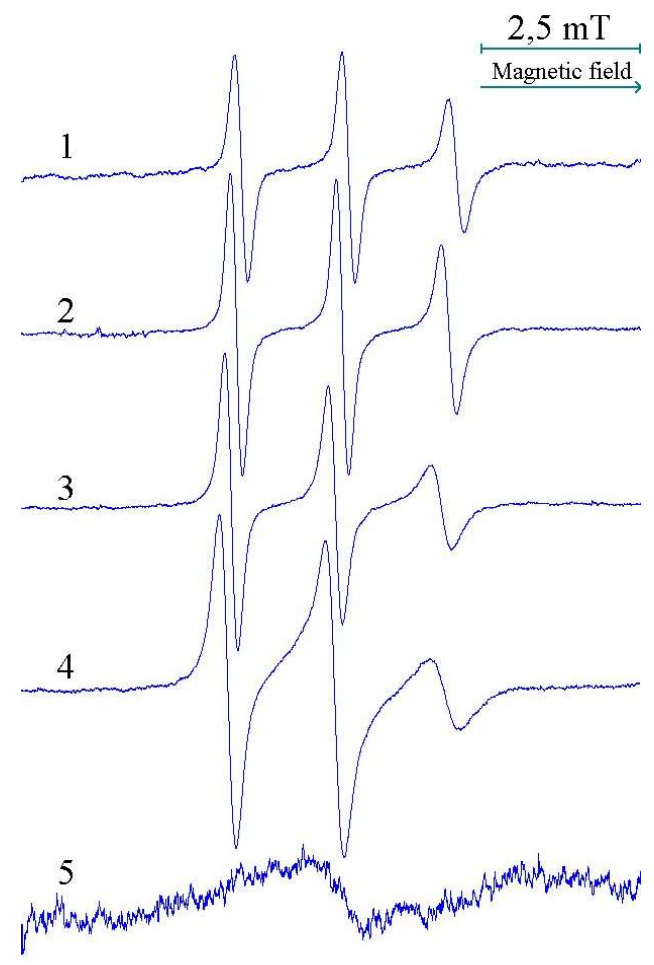

Fig. 2. Probe 2 EPR spectra in: $1-0,5 \% \mathrm{CPC}$ aqueous solution; $2-0,5 \% \mathrm{CPC}$ aqueous solutions containing $15 \% P G$ and $10 \% M 400 ; 3-0,5 \% \mathrm{CPC}$ aqueous solutions containing $15 \% P G, 10 \% M 400$ and $15 \%$

P338; 4 - $17 \%$ P338 aqueous solutions containing $15 \% P G$ and $10 \% M 400 ; 5-15 \% P G$ and $10 \% M 400$ aqueous solution

The EPR spectra of the lipophilic spin probe 2 in aqueous solutions of surfactant are triplets (Fig. 2), which indicates the solubilization of the probe by micelles $[9,24,25]$. In the mixed solvent $\mathrm{PG}-\mathrm{M} 400$ - water (15:10:60) the probe 2 is insoluble, and its EPR spectrum is a singlet (Fig. 2) [9, 24, 25]. In the case of solubilization of probe 2 molecules, the alkyl chain is localized in the nuclei of surfactant micelles, and the nitroxyl radical is localized in the polar part, as evidenced by the values of the isotropic constant $\left(A_{N}\right)$, which were from $1.61 \mathrm{mT}$ to $1.67 \mathrm{mT}$ (Table 2). The information on the packing density and anisotropy of the rotational diffusion of surfactant molecules in the lipophilic nucleus of micelles at the boundary with the polar part was obtained by the parameters of the EPR spectra of probe 2.

As can be seen from Table 2, $15 \%$ $P G$ and $10 \% \mathrm{M} 400$ introduced into the aqueous medium had no effect on the pa- rameters of the EPR spectra of probe 2 in the CPC micelles. Conversely, when P338 was dissolved in the dispersion medium, the rotational correlation times of probe 2 increased, and the parameter $\varepsilon$ became negative. This indicates an increase in the packing density of surfactant molecules in micelles at the interface with the polar part and an increase in their rotational diffusion anisotropy. The higher the P338 concentration in the CPC micellar solution, the greater the values of $\tau_{+1}$ and $\tau_{-1}$. At the P338 content of $15 \%$ the value of $\tau_{+1}$ and $\tau_{-1}$ of probe 2 in the CPC micelles increased by 2.9 and 2.5 times, respectively, in the SLS micelles -3.9 and 3.2 times, and in the MCE micelles - in 1.9 and 2.0 times (Table 2). That is, the interaction of surfactants with P338 led to a change in the structure micelles.

The microviscosity of the nuclei of nonionic surfactant micelles (MCE) at the interface with the polar part was at the level of the microviscosity of liquid paraffin, and the microviscosity of the nuclei of ionic surfactant micelles is approximately 1.6-2.4 times lower (Tables 2,3). In the presence of P338 at a concentration of $15 \%$, the microviscosity of the nuclei of surfactant micelles became close to the microviscosity of white soft paraffin (Tables 2, 3).

P338 in solution also formed micelles that solubilized probe 2. Compared to surfactant micelles, the rotational correlation times $\left(\tau_{ \pm 1}\right.$ and $\left.\tau_{-1}\right)$ of probe 2 in P338 micelles were significantly greater. For example, $\tau_{-1}$ was 6.5 times greater than in the case of SLS, 5.1 times greater than in the case of CPC, and 2.7 times greater than in the case of nonionic surfactant MCE (Table 2).

The physical stability of microheterogeneous disperse systems depends among other things on the viscosity of the liquid dispersion medium, and the release of dissolved active substances from the base-vehicle may depend on the microviscosity of their local surrounding [28].

Table 2

EPR spectra parameters of the spin probe 2

in the micelles of surfactant and P338

\begin{tabular}{|c|c|c|c|c|c|c|c|c|}
\hline \multirow{2}{*}{$\begin{array}{c}\text { Sur- } \\
\text { factant }\end{array}$} & \multicolumn{2}{|c|}{ Content, \% } & \multirow{2}{*}{$\begin{array}{l}\text { Solvent composi- } \\
\text { tion, g }\end{array}$} & \multirow{2}{*}{$\begin{array}{c}\tau_{-1} \cdot 10^{10} \\
\mathrm{~s}\end{array}$} & \multirow{2}{*}{$\begin{array}{c}\tau_{ \pm 1} \cdot 10^{10} \\
\mathrm{~S}\end{array}$} & \multirow{2}{*}{$\varepsilon$} & \multirow{2}{*}{$\begin{array}{l}\Delta H_{0}, \\
\mathrm{mT}\end{array}$} & \multirow{2}{*}{$\begin{array}{l}A_{N}, \\
\mathrm{mT}\end{array}$} \\
\hline & Surfactant & P338 & & & & & & \\
\hline $\mathrm{CPC}$ & 0.5 & 0 & Water & 1.76 & 3.91 & +0.02 & 0.21 & 1.67 \\
\hline CPC & 0.5 & 0 & $\begin{array}{l}\mathrm{PG}-\mathrm{M} 400-\text { wa- } \\
\text { ter }(15: 10: 74.5)\end{array}$ & 1.83 & 3.87 & +0.03 & 0.21 & 1.67 \\
\hline $\mathrm{CPC}$ & 0.5 & 5 & $\begin{array}{c}\text { PG }- \text { M400 - wa- } \\
\text { ter }(15: 10: 69.5)\end{array}$ & 2.08 & 5.52 & -0.12 & 0.21 & 1.63 \\
\hline $\mathrm{CPC}$ & 0.5 & 10 & $\begin{array}{l}\mathrm{PG}-\mathrm{M} 400-\text { wa- } \\
\text { ter }(15: 10: 64.5)\end{array}$ & 3.47 & 9.75 & -0.15 & 0.21 & 1.61 \\
\hline $\mathrm{CPC}$ & 0.5 & 15 & $\begin{array}{l}\mathrm{PG}-\mathrm{M} 400-\text { wa- } \\
\text { ter }(15: 10: 59.5)\end{array}$ & 4.38 & 11.46 & -0.11 & 0.23 & 1.65 \\
\hline SLS & 0.5 & 0 & $\begin{array}{c}\mathrm{PG}-\mathrm{M} 400-\text { wa- } \\
\text { ter }(15: 10: 74.5)\end{array}$ & 1.32 & 3.03 & 0.00 & 0.18 & 1.66 \\
\hline SLS & 0.5 & 15 & $\begin{array}{c}\text { PG }- \text { M400 - wa- } \\
\text { ter }(15: 10: 59.5)\end{array}$ & 4.22 & 11.81 & -0.15 & 0.22 & 1.64 \\
\hline MCE & 0.5 & 0 & $\begin{array}{c}\mathrm{PG}-\mathrm{M} 400-\text { wa- } \\
\text { ter }(15: 10: 74.5)\end{array}$ & 2.63 & 7.26 & -0.12 & 0.22 & 1.62 \\
\hline MCE & 0.5 & 15 & $\begin{array}{c}\mathrm{PG}-\mathrm{M} 400-\text { wa- } \\
\text { ter }(15: 10: 59.5)\end{array}$ & 5.17 & 14.05 & -0.15 & 0.29 & 1.64 \\
\hline - & - & 17 & $\begin{array}{c}\mathrm{PG}-\mathrm{M} 400-\text { wa- } \\
\text { ter }(15: 10: 58)\end{array}$ & 8.94 & 19.69 & -0.02 & 0.29 & 1.69 \\
\hline
\end{tabular}


Table 3

EPR spectra parameters of the spin probe 2 in liquid paraffin and white soft paraffin

\begin{tabular}{|c|c|c|c|c|c|}
\hline Object under study & $\begin{array}{c}\tau_{-1} \cdot 10^{10}, \\
\mathrm{~s}\end{array}$ & $\begin{array}{c}\tau_{ \pm 1} \cdot 10^{10}, \\
\mathrm{~s}\end{array}$ & $\varepsilon$ & $\begin{array}{c}\Delta H_{0}, \\
\mathrm{mT}\end{array}$ & $A_{N}, \mathrm{mT}$ \\
\hline Liquid paraffin & 2.78 & 6.98 & -0.21 & 0.24 & 1.56 \\
\hline White soft paraffin & 4.71 & 13.43 & -0.18 & 0.24 & 1.56 \\
\hline
\end{tabular}

Fig. 3, a shows a plot of dynamic viscosity of aqueous solutions containing $15 \%$ PG and $10 \%$ M400 vice versa the $\mathrm{P} 338$ content. At $25^{\circ} \mathrm{C}$, solutions containing up to $17 \%$ P338 were liquids, and solutions containing more than $18 \%$ P338 were gels. That is, P338 at concentrations of $15-17 \%$ could significantly increase the viscosity of the dispersion medium.

$\underline{\text { Note. As the temperature increases, the } s o l \rightarrow \text { gel }}$ transition occurred at $\mathrm{P} 338$ content which was less than $17 \%$, but these studies are not the subject of this article.

In contrast to the dynamic viscosity of P338 solutions, the value of $\tau_{-1}$ of the probe TEMPON increased linearly in both liquids and creams with increasing P338 content (Fig. 3). This is probably due to the increase in the amount of bound water, rather than the formation of some kind of network causing the change of dynamic viscosity. The sol $\rightarrow$ gel transition in the solution with $20 \%$ P338 did not affect the linear dependence of $\tau_{-1}$ on the P338 content (Fig. 3).

The EPR spectra of the spin probe TEMPON in liquids and creams without P338 and with $17 \%$ P338 are shown in Fig. 4. In addition, the EPR spectrum of the probe TEMPON in $20 \% \mathrm{P} 338$ solution, which was a gel, is provided.

The EPR spectra of the probe TEMPON and the values of $\tau_{-1}$ for both solution and cream with the same P338 content were almost identical and provided evidence that molecules of dissolved probe TEMPON were in a state of rapid isotropic rotation in a liquid medium (Fig. 3, b, 4). But the values of $\tau_{-1}$ of the probe TEMPON in the solution and cream with $17 \%$ P338 were greater by 2.3 and 2.1 times, respectively, compared with the solution and cream without P338 (Fig. 3).

A factor that might affect the rheological properties of creams is the structure of mixed aggregates of surfactants with CSA; the construction of three-dimensional networks and coagulation structures that cause the consistency of the cream are dependent on this structure [9, 10].

Fig. 5 shows the EPR spectra of probe 2 in cream bases with the oil phase and without one (Fig. 5, $a, b$, respectively), with $\mathrm{P} 338$ and without P338 (spectra 1 and spectra 2, respectively) (see the base compositions in Table 4).

The EPR spectra in Fig. 5, $a$ are superpositions of two EPR spectra which are separated in a high-field component. These EPR spectra probably indicate the lateral phase separation in mixed aggregates formed by CPC and CSA. The spectrum with a lower isotropic constant $\left(A_{N}\right)$ indicates the localization of probe 2 in the CPS domains, and spectrum with a higher isotropic constant - in the CSA domains $[9,10]$. When P338 was added into emulsion $\mathrm{o} / \mathrm{w}$ the signal intensity of the probe 2 , localized in the CSA domains, decreased (Fig. 5, a).

Table 4

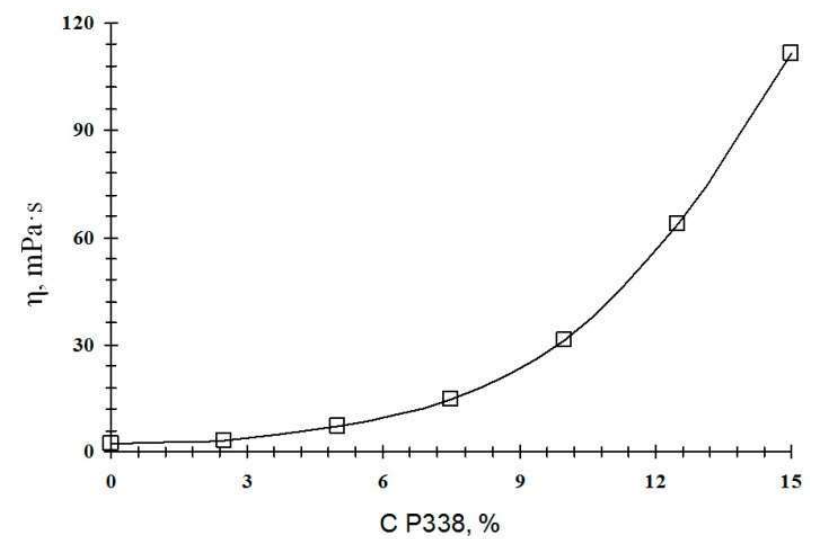

$a$

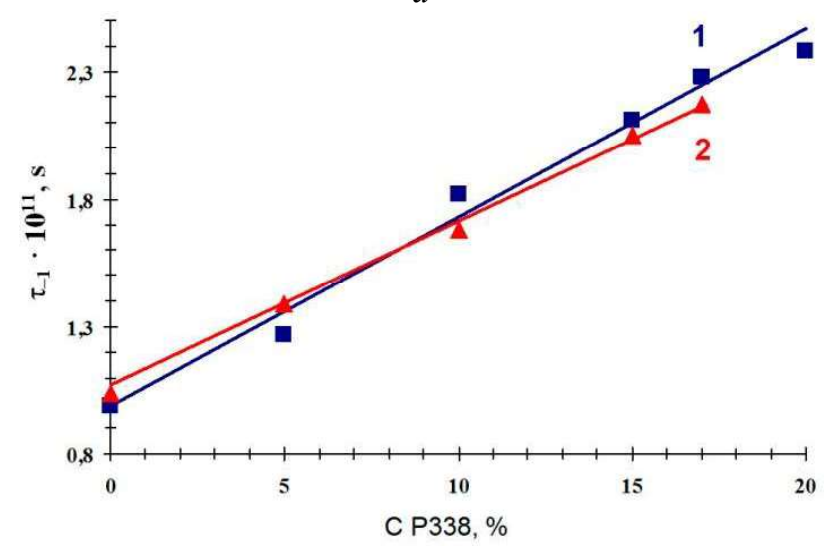

$b$

Fig. 3. $\boldsymbol{a}-\mathrm{P} 338$ content (C) $v s$ dynamic viscosity of P338 aqueous solutions containing $15 \% P G$ and $10 \% M 400$; $\boldsymbol{b}-\mathrm{P} 338$ content $(\mathrm{C}) v s \tau_{-1}$ of spin probe TEMPON in above mentioned solutions (1) and creams based on these solutions with $0.5 \% \mathrm{CPC}$ and 2,0\% CSA (2)

The compositions of the cream bases studied by the spin probes method (Fig. 5), and some parameters of the EPR spectra of the probe 2 in these bases

\begin{tabular}{|c|c|c|c|c|}
\hline \multirow{2}{*}{ Substances } & \multicolumn{4}{|c|}{ Content, ( \% w/w): } \\
\hline & Base 1a & Base 2a & Base $1 b$ & Base $2 b$ \\
\hline $\mathrm{CPC}$ & 0.5 & 0.5 & 0.5 & 0.5 \\
\hline CSA & 4.5 & 4.5 & 2.0 & 2.0 \\
\hline P338 & 0 & 17.0 & 0 & 17.0 \\
\hline PG & 19.0 & 10.0 & 12.5 & 12.5 \\
\hline M400 & 17.0 & 9.0 & 11.2 & 11.2 \\
\hline $\begin{array}{c}\text { Paraffin } \\
\text { Liquid (PL) }\end{array}$ & 15.0 & 15.0 & 0 & 0 \\
\hline Water & 44.0 & 44.0 & 73.8 & 56.8 \\
\hline \multicolumn{5}{|c|}{ Characteristic and parameters of EPR spectra } \\
\hline $\begin{array}{c}\text { Character- } \\
\text { istic }\end{array}$ & \multicolumn{2}{|c|}{\begin{tabular}{|c|} 
Superpositions of 2 \\
triplets
\end{tabular}} & \begin{tabular}{|l|} 
Superposition of 2 \\
triplets and singlet
\end{tabular} & Triplet \\
\hline$A_{N}, \mathrm{mT}$ & $\begin{array}{l}\sim 1.59 \mathrm{i} \\
\sim 2.10\end{array}$ & $\begin{array}{l}\sim 1.59 \mathrm{i} \\
\sim 2.10\end{array}$ & $\sim 1.62 \mathrm{i} \sim 2.10$ & $\sim 1.62$ \\
\hline$\tau_{-1} \cdot 10^{10}, \mathrm{~s}$ & & & & 4.99 \\
\hline$\tau_{+1} \cdot 10^{10}, \mathrm{~s}$ & & & & 13.27 \\
\hline$\varepsilon$ & & & & -0.18 \\
\hline$\Delta H_{0}, \mathrm{mT}$ & 0.38 & 0.38 & 0.33 & 0.23 \\
\hline
\end{tabular}



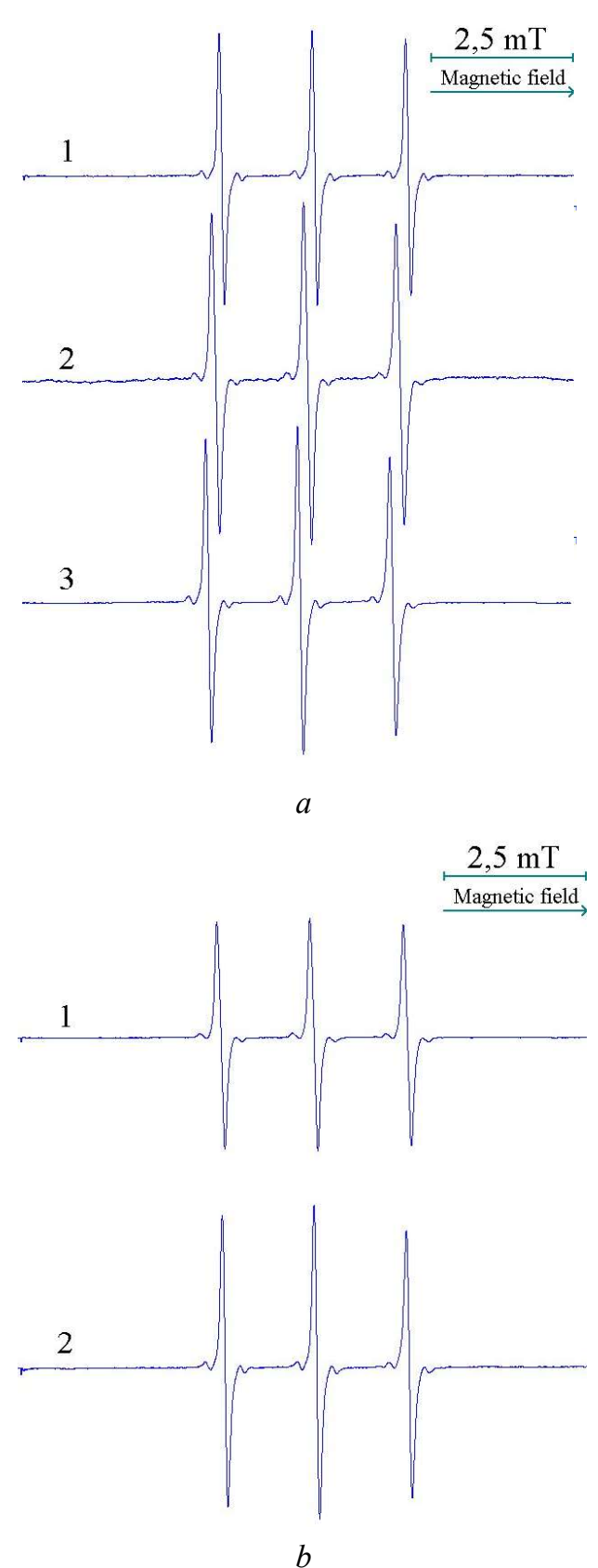

Fig. 4. EPR spectra of the spin probe TEMPON in: $\boldsymbol{a}$-solutions: without P338 (1); with 17 \% P338 (2) and with $20 \%$ P338 (3); b - creams: without P338 (1) and with $17 \%$ P338 (2)

Note. The composition of the solutions and creams see in the captions to Fig. 3

The EPR spectrum No. 1 of probe 2 at the base $\mathbf{1 b}$ is a superposition of three EPR spectra: two triplets and a singlet (Fig. 5, $b$ ). Triplets with $A_{N} 1.62 \mathrm{mT}$ and $2.10 \mathrm{mT}$ indicate the localization of probe 2 in the CPC domains and CSA domains, and the singlet indicates the association of radicals in the domains with higher packing density of CSA molecules. Addition of $17 \%$ P338 into the disperse system without oil phase (base $2 \boldsymbol{b}$ ) led to the conversation of the EPR spectrum into a triplet (Fig. 5, b), which indicate a uniform distribution of probe 2 molecules in the phase of their localization. The microviscosity of this phase was approximately the same as that of white soft paraffin, as evidenced by the close values of $\tau_{-1}$ and $\tau_{ \pm 1}$ (Tables 3,4). Compared with CPC micelles, the values of $\tau_{-1}$ and $\tau_{+1}$ in this phase were 1.6 and 3.4 times greater, respectively. They were close to the values of $\tau_{-1}$ and $\tau_{ \pm 1}$, calculated from the EPR spectra of probe 2 in $0.5 \%$ CPC solution containing $15 \%$ P338. The anisotropy parameters of the EPR spectra of probe 2 in this solution $(\varepsilon=-0.15)$ and in base $\mathbf{2 b}(\varepsilon=-0.18)$ were also similar (Tables 2, 4). The nitroxyl radical of probe 2 in the base $\mathbf{2} \boldsymbol{b}$ was in contact with the polar medium $\left(A_{N}=1.62 \mathrm{mT}\right)$.

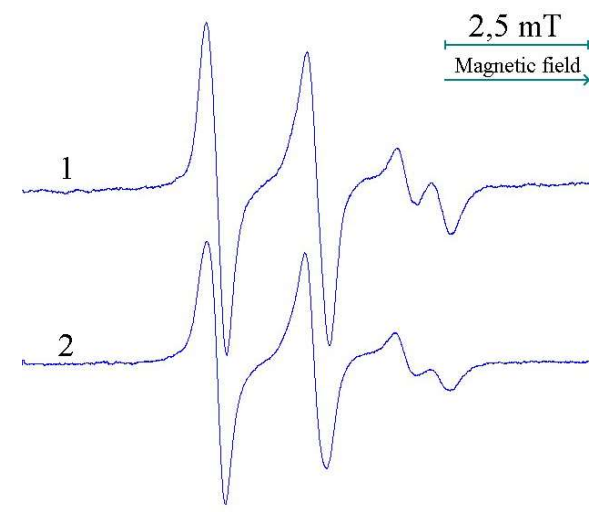

$a$

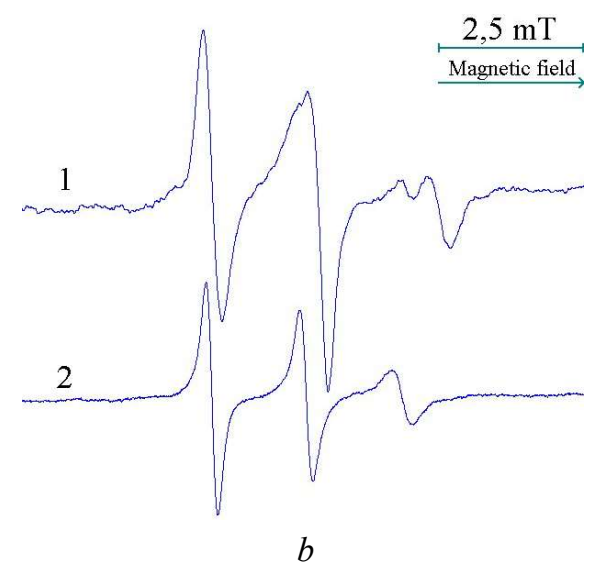

Fig. 5. EPR spectra of spin probe 2 in cream bases $\mathbf{1} a$, $\mathbf{2} \boldsymbol{a}, \mathbf{1} \boldsymbol{b}$ and $\mathbf{2 b}$ (cream base compositions are provided in Table 4)

The EPR spectra of probes 1, 3 and 4 in the case of base $\mathbf{1} \boldsymbol{b}$ without P338 were also superpositions (Fig. 6, a). The EPR spectrum of probe 1 was a superposition of two EPR spectra separated in a high-field component; the EPR spectrum of probe 3 was a superposition of the triplet and singlet, and the EPR spectrum of probe 4 was a superposition of two spectra, one of which was anisotropic. This indicates a lateral phase separation in the aggregates formed by CPC and CSA, along the entire length of alkyl chains.

The EPR spectra of probes 1, 3 and 4 based on fatty acids in base $\mathbf{2} \boldsymbol{b}$ with $17 \% \mathrm{P} 338$, on the contrary, were triplets (Fig. 6, b), which indicates a uniform distribution of surfactant molecules along the length of alkyl chains in phase of their localization. The EPR spectra parameters of probes 1, 2, 3 and 4 in the case of base $\mathbf{2 b}$ are presented in Table 5. They depended not only on the structure of surfactant aggregates, but also on the molec- 
ular structure of a particular spin probe. But it is obvious that the free radical of each of the probes was localized in a certain part of the surfactant aggregates because the isotropic constants $\left(A_{N}\right)$ for them differed significantly from $1.64 \mathrm{mT}$ to $1.46 \mathrm{mT}$ and indicated a polar and hydrophobic environment of radicals, respectively (Table 5).

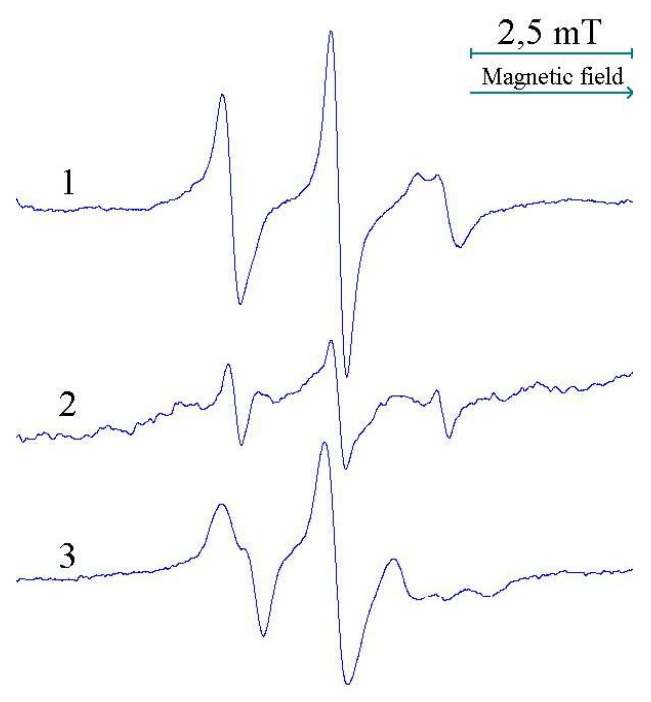

$a$

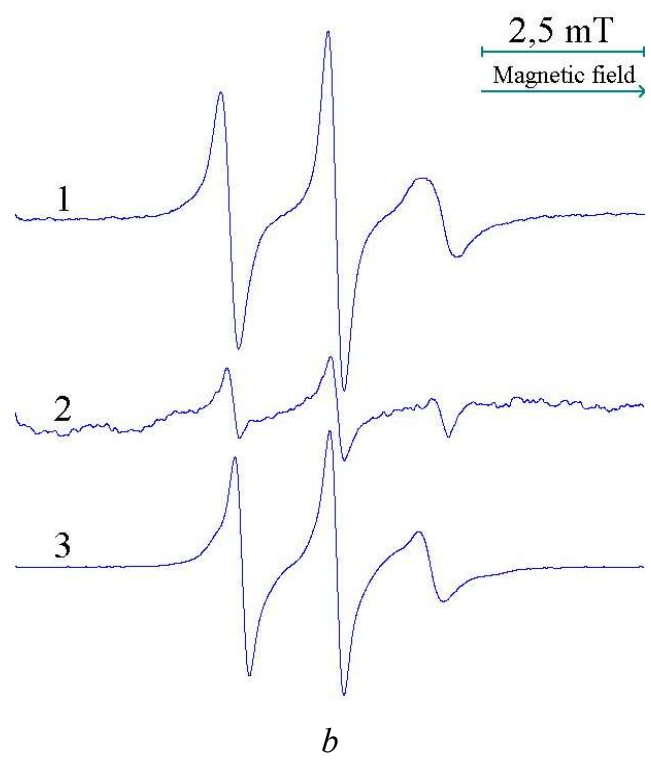

Fig. 6. EPR spectra of the probes 1 (1), 3 (2) and 4 (3) in the cream bases $\mathbf{1} \boldsymbol{b}$ without P338 (a) and $\mathbf{2 b}$ with $17 \%$ P338 (b) (Table 4)

Table 5

Parameters of EPR spectra of the spin probes 1, 2, 3 and 4 in the cream base $2 \boldsymbol{b}$

\begin{tabular}{|c|c|c|c|c|c|c|}
\hline $\begin{array}{c}\text { Spin } \\
\text { probe }\end{array}$ & $\begin{array}{c}\tau_{+1} \cdot 10^{10}, \\
\mathrm{~s}\end{array}$ & $\begin{array}{c}\tau_{-1} \cdot 10^{10}, \\
\mathrm{~s}\end{array}$ & $\begin{array}{c}\tau_{ \pm 1} \cdot 10^{10}, \\
\mathrm{~s}\end{array}$ & $\varepsilon$ & $\begin{array}{c}\Delta H_{0}, \\
\mathrm{mT}\end{array}$ & $\begin{array}{c}A_{N}, \\
\mathrm{mT}\end{array}$ \\
\hline Probe 1 & 24.16 & 8.77 & 14.96 & +0.16 & 0.27 & 1.64 \\
\hline Probe 2 & & 4.99 & 13.27 & -0.18 & 0.23 & 1.62 \\
\hline Probe 3 & 24.77 & 3.91 & 4.48 & +0.35 & 0.21 & 1.58 \\
\hline Probe 4 & 11.80 & 5.95 & 11.48 & +0.11 & 0.23 & 1.46 \\
\hline
\end{tabular}

According to the results of research by spin probes method it was shown that P338 changed the structure of aggregates formed by CPC and CSA, both in emulsions $\mathrm{o} / \mathrm{W}$ and in cream bases without oil phase. It was interesting to study the impact of these changes on the microstructure, rheological parameters and functional properties of the bases.

Study of the effect of P338 on the properties of cream bases. Rheological properties, microstructure, adhesion and water absorption

The rheograms of cream bases without oil phase at different contents of P388 are shown in Fig. 7, and rheological parameters of these bases at $25^{\circ} \mathrm{C}$ are provided in Table 6.

The apparent viscosity of the bases increased by about 4-6 times with an increase in the content of P338 up to $15 \%$ (Table 6 ), which can be explained by increasing the viscosity of the dispersion medium (Fig. 3,a). The bases were characterized by plastic flow with insignificant thixotropic properties (Fig. 7).

When the $\mathrm{P} 338$ content is $17 \%$ the flow behaviour of the base changed, and its rheological parameters increased significantly. Compared with the base without P338, the yield stress $\left(\tau_{0}\right)$ increased 10.8 times, the hysteresis area $\left(A_{H}\right)-5.6$ times, and the apparent viscosity $(\eta)$ at low shear rates $\left(D_{r}\right)$ - more than 8 times.

The bases with P338 had a yield stress as systems with a plastic flow, but at the particular shear rates the flow behaviour became pseudoplastic; at exposure of shear stress the structure of the base was ruined, which are illustrated by the large hysteresis areas (Fig. 7, 8).

Table 6

Rheological parameters of cream bases of type $\boldsymbol{a}$ and type $\boldsymbol{b}$ with different contents of P338

\begin{tabular}{|c|c|c|c|c|c|c|c|}
\hline \multirow[b]{2}{*}{$\begin{array}{l}\text { Base } \\
\text { type }\end{array}$} & \multirow[b]{2}{*}{ Surfactant } & \multirow{2}{*}{$\begin{array}{c}\text { C P338, } \\
\%\end{array}$} & \multirow[b]{2}{*}{$\tau_{0}, \mathrm{~Pa}$} & \multirow{2}{*}{$\left|\begin{array}{c}A_{H} \\
\mathrm{~Pa} \cdot \mathrm{s}^{-1}\end{array}\right|$} & \multicolumn{3}{|c|}{$\eta, \mathrm{Pa} \cdot \mathrm{s}^{-1}$ at $D_{r}$ : } \\
\hline & & & & & $\begin{array}{c}14.59 \\
\mathrm{~s}^{-1}\end{array}$ & \begin{tabular}{|c|}
41.66 \\
$\mathrm{~s}^{-1}$
\end{tabular} & $\begin{array}{c}82.31 \\
\mathrm{~s}^{-1}\end{array}$ \\
\hline$b$ & \multirow{7}{*}{$\begin{array}{l}\text { CPC } 0.5 \%+ \\
+ \text { CSA } 2.0 \%\end{array}$} & 0 & 18.7 & 1497 & 2.26 & 0.85 & 0.50 \\
\hline$b$ & & 3 & 32.9 & 1002 & 3.31 & 1.28 & 0.78 \\
\hline$b$ & & 7 & 62.8 & 346 & 5.73 & 2.38 & 1.55 \\
\hline$b$ & & 11 & 58.7 & 475 & 6.24 & 3.03 & 2.18 \\
\hline$b$ & & 13 & 60.2 & 927 & 7.44 & 3.91 & 2.71 \\
\hline$b$ & & 15 & 76.9 & 1665 & 9.89 & 4.77 & 3.31 \\
\hline$b$ & & 17 & 201.1 & 8436 & 19.41 & 7.01 & 3.30 \\
\hline$b$ & \multirow{2}{*}{$\begin{array}{l}\text { Kolliphor }{ }^{\circledR} \\
\text { CSL 2,5 \% }\end{array}$} & 0 & 19.8 & 895 & 2.13 & 0.91 & 0.60 \\
\hline$b$ & & 17 & 193.6 & 12376 & 17.39 & 4.92 & 2.05 \\
\hline$b$ & \multirow{2}{*}{$\begin{array}{l}\text { MCE } 0.5 \%+ \\
+ \text { CSA } 2.5 \%\end{array}$} & 0 & 10.61 & 928 & 1.19 & 0.45 & 0.24 \\
\hline$b$ & & 17 & 219.3 & 6933 & 18.91 & 6.92 & 3.40 \\
\hline$a$ & \multirow{2}{*}{$\begin{array}{l}\text { CPC } 0.5 \%+ \\
+ \text { CSA } 4.5 \%\end{array}$} & 0 & 104.8 & 3866 & 11.42 & 4.68 & 2.87 \\
\hline$a$ & & 17 & 641.1 & 31480 & 55.81 & 22.72 & 12.76 \\
\hline
\end{tabular}

Such changes in flow behaviour and rheological parameters caused by $17 \%$ P338 added into dispersed systems occurred with cream bases (without oil phase) with anionic surfactant and nonionic surfactant used as emulsifiers $\mathrm{o} / \mathrm{w}$, and also in the case of emulsions $\mathrm{o} / \mathrm{w}$ with $15 \%$ oil phase (Table 6 , Fig. 8). 

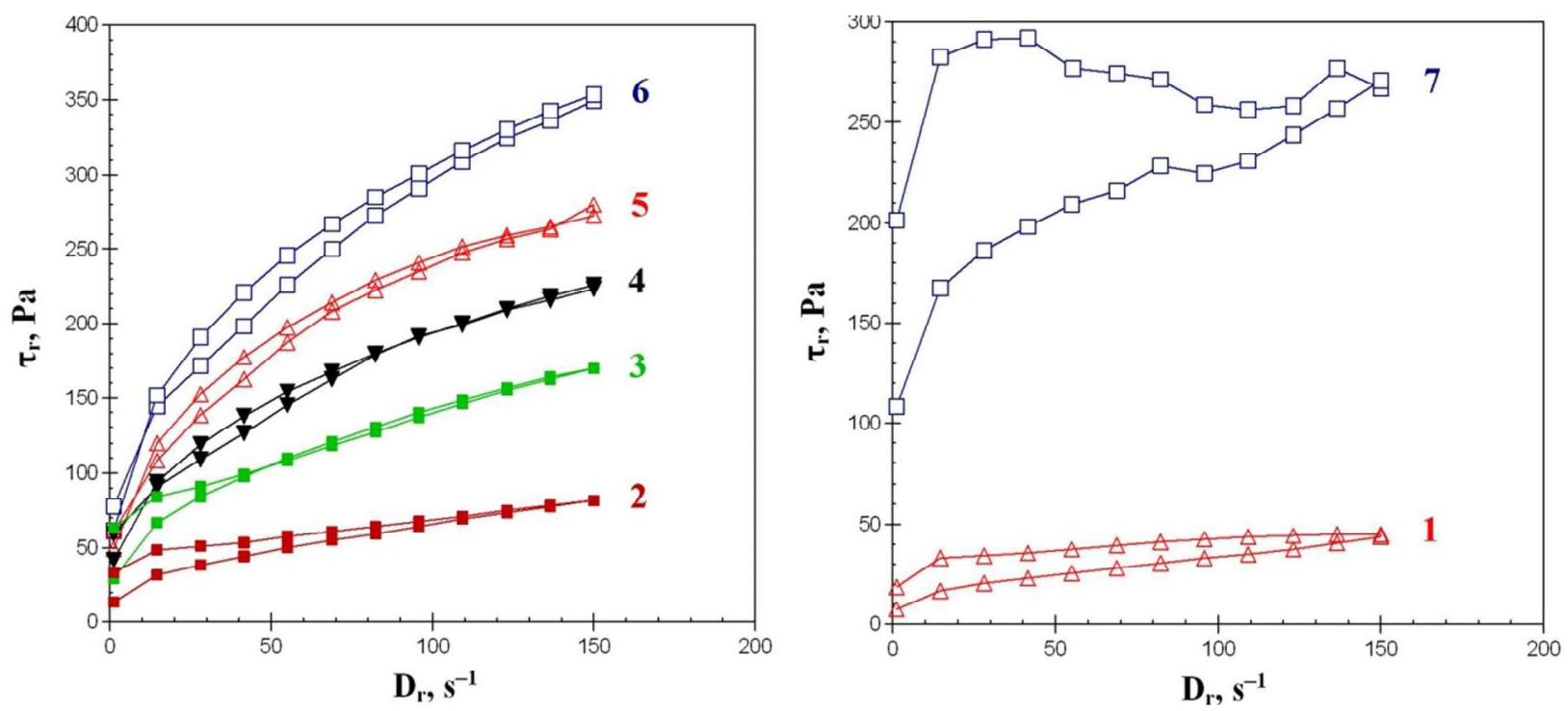

Fig. 7. Rheograms of the cream bases of type $\boldsymbol{b}$ (without oil phase) at P388 content: $1-0 \%, 2-3 \% ; 3-7 \% ; 4-$ $11 \% ; 5-13 \% ; 6-15 \% ; 7-17 \%$
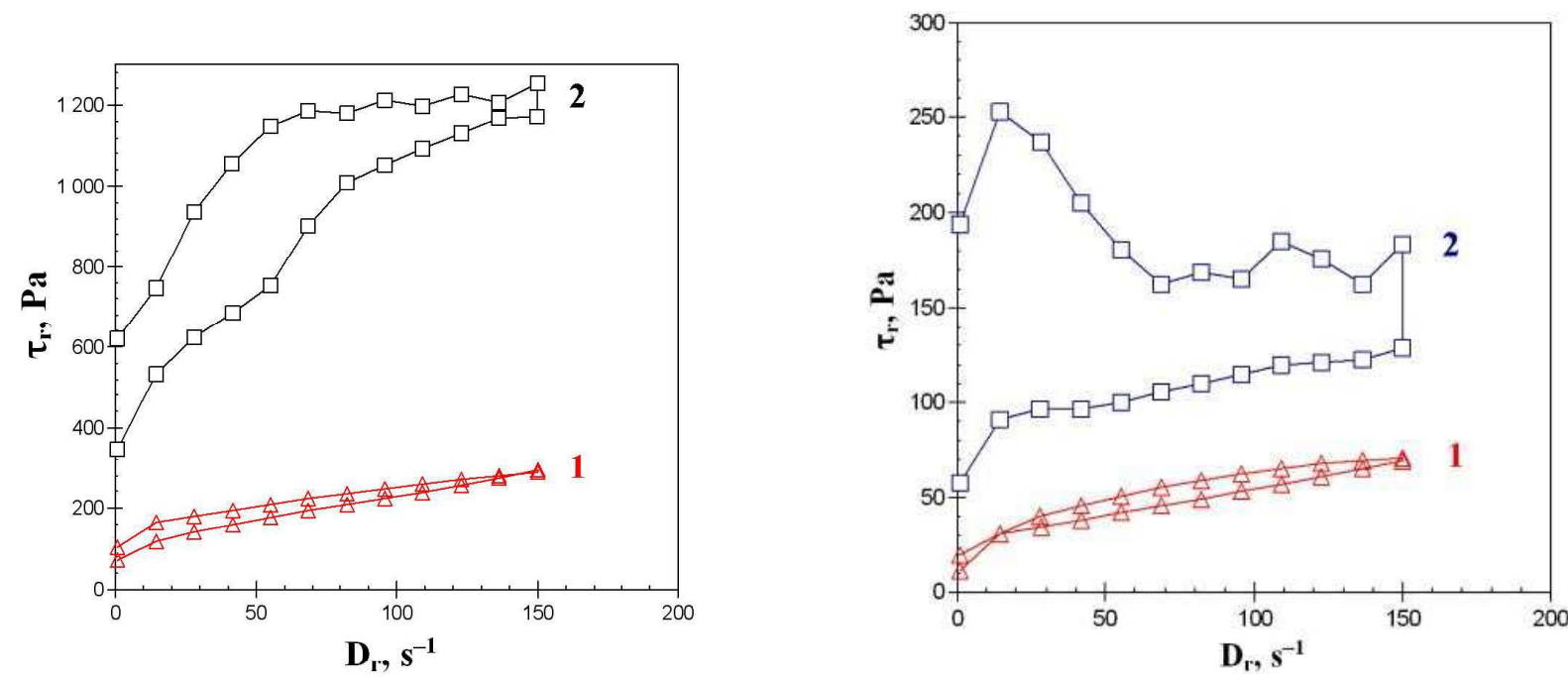

$a$

$b$

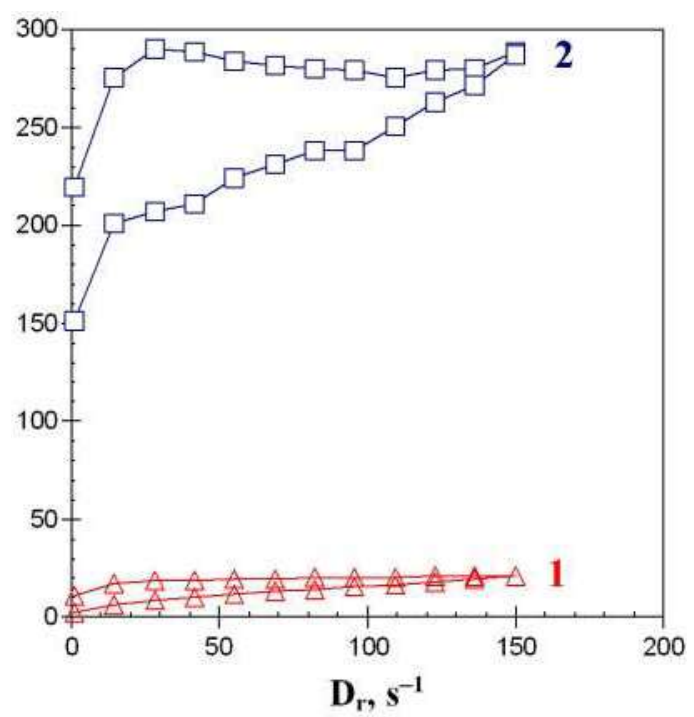

$c$

Fig. 8. Rheograms of cream bases with oil phase $(\boldsymbol{a})$ and without oil phase $(\boldsymbol{b}, \boldsymbol{c})$, containing cationic surfactant $(\boldsymbol{a})$, anionic surfactant (b) and nonionic surfactant (c) without P338 (1) and with 17 \% P388 (2) 
The micrographs of cream bases $\mathbf{1} \boldsymbol{b}$ and $\mathbf{2} \boldsymbol{b}$ without oil phase are provided in Fig. 9; these bases were colloidal dispersions of two emulsifiers $\mathrm{o} / \mathrm{w}$ and $\mathrm{w} / \mathrm{o}$ : $\mathrm{CPC}$ and CSA, respectively.

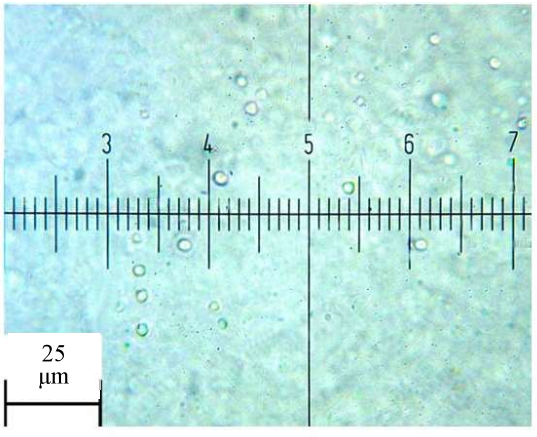

$1 b$

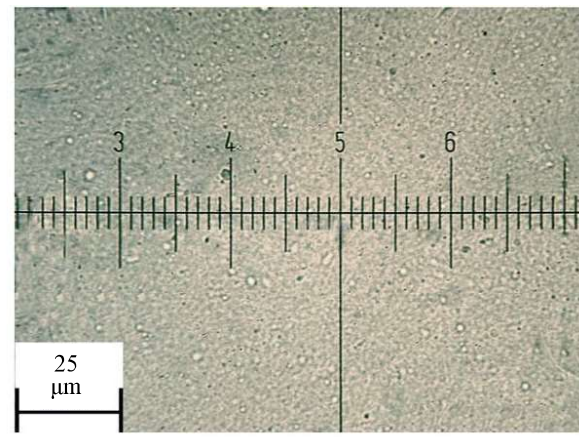

$2 b$
Fig. 9. Micrographs of the bases $\mathbf{1 b}$ and $\mathbf{2} \boldsymbol{b}$ (Table 4); at $\times 600$
In the presence of $17 \% \mathrm{P} 338$ in the base, a structured system with high apparent viscosity was formed at $70{ }^{\circ} \mathrm{C}$ (Fig. 10). In the temperature range from $55^{\circ} \mathrm{C}$ to $25^{\circ} \mathrm{C}$, the apparent viscosity was approximately the same. In the case of the base without P338, the apparent viscosity of such base decreased with increasing temperature, at about $55^{\circ} \mathrm{C}$ the base melted and became a Newtonian fluid (Fig. 10).

Since bases without P338 and with P338 had thixotropic properties, change of apparent viscosity, which reflects destruction or restoration of the coagulation structure, was studied during stirring the base at rotational speed of $30 \mathrm{rpm}$ and then keeping without mixing. Table 7 provides the data obtained.

In the micrograph of the base $\mathbf{1 b}$ without P338 there are no particles of the dispersed phase formed by the emulsifiers (only a few air bubbles formed during preparation the sample are visible). In such disperse system, emulsifiers form mixed aggregates that are lyotropic liquid crystals. In the dispersion medium, a three-dimensional network is formed from the emulsifier aggregates, due to which a coagulation structure is formed. It provides certain rheological parameters of the dispersed system, which is a non-Newtonian fluid (see rheogram 1 in Fig. 7). It is the lyotropic liquid crystals where the lateral phase separation takes place, which is detected by the spin probes method (Fig. 5, 6).

In the micrograph of the base $2 \boldsymbol{b}$ with $17 \% \mathrm{P} 338$, on the contrary, there are numerous particles of the dispersed phase with sizes from about $0.5 \mu \mathrm{m}$ to $2 \mu \mathrm{m}$. Probably, this dispersed phase was cetostearyl alcohol (CSA). Due to the interaction of cationic surfactant (CPC) with P338, lyotropic liquid crystals, which should have contained a mixture of CPC and CSA, were not formed. It can be assumed that CPC together with P338 were adsorbed on the surface of dispersed CSA particles, and P338, dissolved in the dispersion medium, «crosslinked» individual particles of the dispersed phase with adsorption layers by ion-dipole and steric interactions with CPC. With a sufficient content of P338, this three-dimensional network was formed through the entire volume of the dispersed system, which led to a change in the flow behaviour. It could be assumed that a significant increase in the rheological parameters of the base with $17 \% \mathrm{P} 338$ is associated with the formation of a gel in the dispersion medium. But this factor would not affect the structure of the aggregates of CPC with CSA and the microstructure of base, which converted into the suspension of CSA.

The formation of a very viscous dispersed system occurred at $65-70{ }^{\circ} \mathrm{C}$ after mixing degassed phases A and $\mathrm{B}$ under vacuum (section 3). The dependence of the apparent viscosity of the cream base on temperature are illustrated in Fig. 10 stress the decrease of their apparent viscosity occurred, which was the greatest within 2 min after start of experiment. Then till the $30^{\text {th }}$ minute, the apparent viscosity of the base $1 \boldsymbol{b}$ slowly decreased from $0.80 \mathrm{~Pa} \cdot \mathrm{s}$ to $049 \mathrm{~Pa} \cdot \mathrm{s}$, approximately the same. After 30 minutes of stirring, the apparent viscosity of base $1 \boldsymbol{b}$ decreased by $65.74 \%$ and the apparent viscosity of base $2 \boldsymbol{b}$ by $59.73 \%$.

After keeping without stirring for $24 \mathrm{~h}$, the apparent viscosity reached up to $79.72 \%$ of the initial value for base $\mathbf{1} \boldsymbol{b}$ and $105.88 \%$ for base $\mathbf{2 b}$. That is, stirring during production process should be considered as critical in the case of base $1 b$ taking into account its low initial apparent viscosity. The apparent viscosity of base $\boldsymbol{2} \boldsymbol{b}$ was increasing significantly sooner, as it recovered from $40.27 \%$ to $63.19 \%$ within $1 \mathrm{~h}$ and up to $67.87 \%$ within $2 \mathrm{~h}$ (Table 7).

Table 7

Change of apparent viscosity $(\eta)$ of cream bases $\mathbf{1 b}$ and $\boldsymbol{2} \boldsymbol{b}$ (Table 4 ) while stirring at $30 \mathrm{rpm}$ and then after keeping without stirring

\begin{tabular}{|c|c|c|c|c|}
\hline \multirow{2}{*}{ Time } & \multicolumn{2}{|c|}{ Cream base $\mathbf{1 b}$} & \multicolumn{2}{c|}{ Cream base $\mathbf{2 b}$} \\
\cline { 2 - 5 } & $\eta, \mathrm{Pa} \cdot \mathrm{s}$ & $\%$ & $\eta, \mathrm{Pa} \cdot \mathrm{s}$ & $\%$ \\
\hline $\begin{array}{c}\text { 0 (initial } \\
\text { value) }\end{array}$ & 1.43 & 100.00 & 6.63 & 100.0 \\
\hline \multicolumn{5}{|c|}{ during stirring } \\
\hline 1 min & 0.87 & 60.83 & 2.91 & 43.89 \\
\hline $2 \mathrm{~min}$ & 0.80 & 55.94 & 2.58 & 38.91 \\
\hline $3 \mathrm{~min}$ & 0.75 & 52.44 & 2.66 & 40.12 \\
\hline $4 \mathrm{~min}$ & 0.72 & 50.34 & 2.57 & 38.76 \\
\hline $5 \mathrm{~min}$ & 0.70 & 48.95 & 2.59 & 39.06 \\
\hline $10 \mathrm{~min}$ & 0.60 & 41.95 & 2.70 & 40.72 \\
\hline $15 \mathrm{~min}$ & 0.55 & 38.46 & 2.64 & 39.81 \\
\hline $20 \mathrm{~min}$ & 0.52 & 36.36 & 2.62 & 39.51 \\
\hline $30 \mathrm{~min}$ & 0.49 & 34.26 & 2.67 & 40.27 \\
\hline \multicolumn{5}{|c|}{ after keeping without stirring } \\
\hline $1 \mathrm{~h}$ & 0.50 & 34.96 & 4.19 & 63.19 \\
\hline $2 \mathrm{~h}$ & 0.52 & 36.36 & 4.50 & 67.87 \\
\hline $24 \mathrm{~h}$ & 1.14 & 79.72 & 7.02 & 105.88 \\
\hline
\end{tabular}

When both of tested bases were exposed to shear but the apparent viscosity of the base $\boldsymbol{2} \boldsymbol{b}$ continued to be 


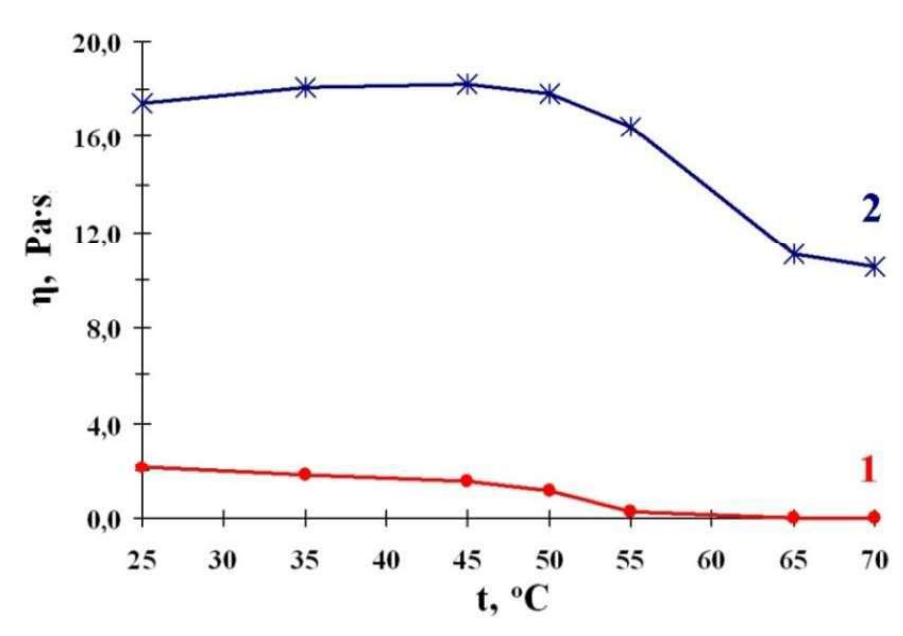

Fig. 10. The apparent viscosity $(\eta)\left(D_{r}=14.59 \mathrm{~s}^{-1}\right) v s$ temperature for the cream base (containing $2.5 \%$ Kolliphor $^{\circledR}$ CSL and not containing the oil phase) without P338 (1) and with $17 \%$ P388 (2)

\section{Adhesion}

Table 8 provides the results of assessment of adhesion by pull-off test for $15 \%$ P338 aqueous solution and $17 \%$ P338 aqueous solution, which additionally contained $12.5 \% \mathrm{PG}$ and $11.2 \% \mathrm{M} 400$, as well as for cream bases $\mathbf{1} \boldsymbol{b}$ and $\mathbf{2} \boldsymbol{b}$ (Table 4 ).

According to Table 8, with increasing P338 content from $15 \%$ to $17 \%$ the strength of adhesion increased by 2 times. Strength of adhesion in the case of cream base $2 \boldsymbol{b}$ with $17 \%$ P338 was 3.5 times greater than in the case of cream base $\mathbf{1 b}$ without P338. Thus, it was P338 which mainly provided adhesion in the case of this base. It should be noted that among objects under study, the hydrophilic base $\mathbf{2} \boldsymbol{b}$ had the highest apparent viscosity together with the greatest strength of adhesion. The combination of such properties is optimal for creams intended, for example, for vaginal use.

\begin{tabular}{|c|c|c|c|}
\hline \multicolumn{4}{|c|}{$\begin{array}{c}\text { Table } 8 \\
\text { Strength of adhesion }\left(S_{m}\right) \text { of P338 solutions and } \\
\text { cream bases } \mathbf{1 b} \text { and } \mathbf{2 b}\end{array}$} \\
\hline \multicolumn{4}{|c|}{$S_{m}\left(\mathrm{~kg} / \mathrm{sm}^{2}\right)$ in the case of: } \\
\hline $\begin{array}{c}15 \% \text { P338 } \\
\text { solution }\end{array}$ & $\begin{array}{c}17 \% \mathrm{P} 338 \\
\text { solution }\end{array}$ & $\begin{array}{c}\text { Cream base } \\
\qquad \mathbf{1 b}\end{array}$ & $\begin{array}{c}\text { Cream base } \\
\qquad \boldsymbol{2 b}\end{array}$ \\
\hline 0.066 & 0.134 & 0.044 & 0.154 \\
\hline
\end{tabular}

\section{Osmotic activity (water absorption)}

The difference in osmotic pressure between water and cream or gel or liquid, separated by a permeable membrane, causes multidirectional diffusion processes:

1) active substances and some excipients release into the chamber with water;

2) water diffuses into a chamber with cream, gel or liquid [28].

The molecular mass of P338 is established from 12,700 to 17,400 [1], therefore P338 molecules are not able to diffuse through the permeable membrane. But solution of P338 might absorb water through a membrane. The ability to absorb water is an important property of topical preparation, because, on the one hand, it can provide exudate absorption and contribute to the therapeutic effect, and, on the other hand, can cause tissue dehydration and local irritation [6].

The results of the study of water absorption by $17 \%$ P338 solution, which was a liquid (1), by $20 \%$ P338 solution, which was a gel (2), and by cream base $2 \boldsymbol{b}$ (3) containing $17 \%$ P338 in dissolved state (see Table 4) are presented in Fig. 11 and Table 9.

The increase in $\mathrm{P} 338$ content in solutions from $17 \%$ to $20 \%$ and sol $\rightarrow$ gel transition, resulting from it, had little effect on parameters of water absorption. Thus, the rate of water absorption (R) by gel (2) increased by an average of $23.47 \%$, and the cumulative amount of absorbed water compared to the liquid (1) increased by an average of $15.85 \%$ due to an increase in content of P338 from $17 \%$ up to $20 \%$. That is, the structural sol $\rightarrow$ gel transition did not lead to a decrease in the rate of water absorption and cumulative amount of absorbed water. On the contrary, the change in the microstructure in the case of base $\mathbf{2} \boldsymbol{b}$, which also contained $17 \%$ P338, led to a significant decrease in parameters of water absorption (Table 9). The rate of water absorption (R) by the base $2 \boldsymbol{b}$ (3) decreased by an average of 7.63 times, and the cumulative amount of absorbed water compared to the liquid (1) decreased by an average of 6.55 times.

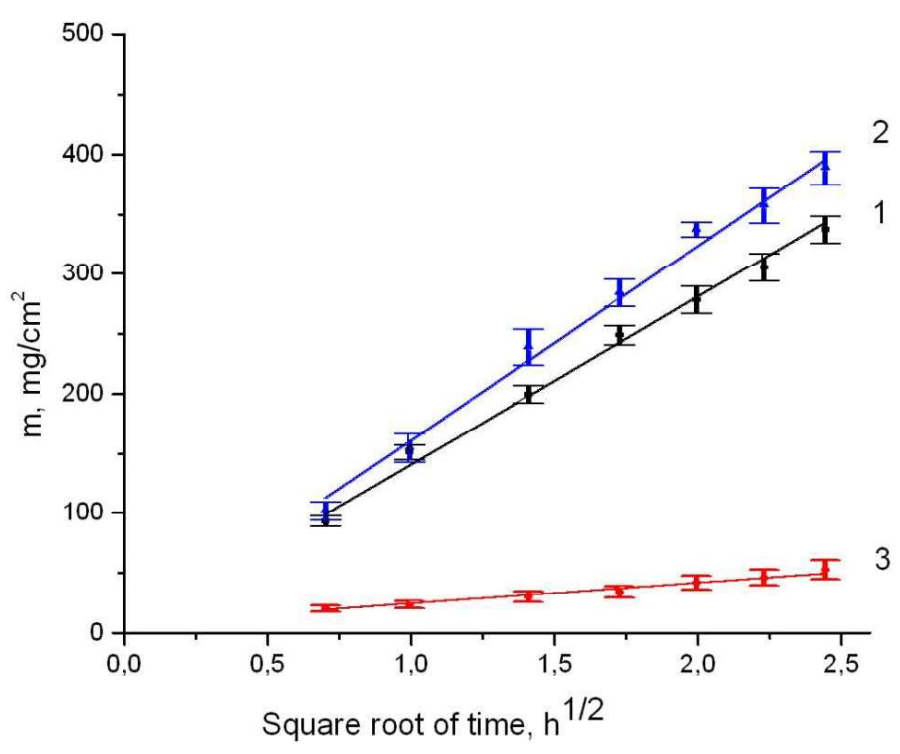

Fig. 11. Water absorption plots for liquid (1), gel № 1 (2) and base $\mathbf{2 b}(\mathbf{3})$

The research results show that significant changes in the properties of base $\mathbf{2} \boldsymbol{b}$ (increase in rheological parameters and decrease in water absorption) are primarily caused by the formation of the three-dimensional network due to the interaction of $\mathrm{P} 338$ with adsorption layers on the particles of the dispersed phase formed by CSA. 
Water absorption parameters by liquid (1), gel (2) and cream base $\mathbf{2 b}(\mathbf{3})$

\begin{tabular}{|c|c|c|c|}
\hline \multirow{2}{*}{ Parameter } & \multicolumn{3}{|c|}{ Results } \\
\cline { 2 - 4 } & Liquid (1) & Gel (2) & Base 2b (3) \\
\hline \multirow{2}{*}{ Release rate $(R), \mu \mathrm{g} / \mathrm{cm}^{2} / \mathrm{h}^{-1 / 2}$} & $134.96 \pm 10.03$ & $166.63 \pm 12.36$ & $17.68 \pm 3.33$ \\
& SD: 4.98 & SD: 6.13 & 51.65 \\
\hline $\begin{array}{c}\text { Cumulative amount }(\mathrm{A}) \text { of absorbed water } \\
\text { (at the time point } 6 \mathrm{~h}), \mu \mathrm{g} / \mathrm{cm}^{2}\end{array}$ & $337.35 \pm 22.72$ & $390.68 \pm 19.84$ & SD: 4.17 \\
\hline $\mathrm{A}_{2 / 3} / \mathrm{A}_{1}, \%$ & SD: 11.27 & $107.96-125.98$ & $13.21-17.91$ \\
\hline$R_{2 / 3} / R_{1}, \%$ & - & $113.43-135.57$ & $11.44-16.08$ \\
\hline Correlation coefficient r & - & $0.99502 \pm 0.00243$ & $0.99334 \pm 0.00484$ \\
\hline Coefficient of determination $\mathrm{R}^{2}$ & $0.99649 \pm 0.00354$ & SD: 0.00120 & SD: 0.00240 \\
\hline \multirow{2}{*}{ Cumulative amount of water $($ after $6 \mathrm{~h}), \%$} & SD: 0.00176 & 0.990 & 0.987 \\
\hline
\end{tabular}

\section{Discussion of research results}

Under the impact of poloxamer 338 in aqueous solutions of surfactants (cationic surfactant CPC, anionic surfactant SLS and nonionic surfactant MCE) the hydrodynamic diameter of the micelles and the absolute values of $\zeta$-potential decrease (Table 1). These phenomena are probably due to the formation mixed micelles of surfactants and P338. The hydrodynamic diameter of the micelles are due to the surfactants that prevent the formation of larger micelles of P338 (Table 1, Fig. 1). It was shown by spin probes method that as the P338 concentration increases, both the packing density of surfactant molecules in micelle nuclei at the interface with the polar part and the anisotropy of their rotational diffusion increase (Table 2). That is, the interaction of surfactants with P338 leads to changes in the structure of micelles. According to the parameters of rotational diffusion of spin probes based on fatty acids, the nuclei of surfactant micelles are two-dimensionally liquid at $25{ }^{\circ} \mathrm{C}$ (Table 2, Fig. 2) [29]. In the presence of $15 \%$ P338, the microviscosity of the micelle nuclei becomes similar to the microviscosity of white soft paraffin (Tables 2,3 ).

Poloxamer 338 in solutions forms micelles [23] that solubilize lipophilic probe 2 , but in these micelles the rotational correlation times of probe 2 are greater than in the micelles of CPC, SLS and MCE (Table 2, Fig. 2). As the $\mathrm{P} 338$ concentration increases, the dynamic viscosity of the solutions which are the dispersion medium of cream bases rises (Fig. 3, a). At $25^{\circ} \mathrm{C}$, solutions containing up to $17 \%$ P338 are liquids, and solutions containing more than $18 \%$ P338 become gels [23]. P338 binds water in solutions, because of which the rotational correlation time $\left(\tau_{-1}\right)$ of the TEMPON hydrophilic probe increases linearly with increasing P338 content both in liquids and in the dispersion medium of cream bases (Fig. $3, b$ ).

A factor that might affect the rheological properties of creams is the structure of mixed aggregates of surfactants with CSA; the construction of three-dimensional networks and coagulation structures that cause the consistency of the cream are dependent on this structure $[9,10]$. According to the results of research by spin probes method, P338 changes the structure of aggregates formed by CPC and CSA, both in emulsions o/w and in cream bases without an oil phase (Tables 4, 5; Fig. 5, 6).
With the increase in the content of P338 up to $15 \%$ inclusive cream bases are characterized by plastic flow with insignificant thixotropic properties and their apparent viscosity increases by several times (Fig. 7, Table 6) that might be explained by increasing the viscosity of the dispersion medium (Fig. 3,a). When the P338 content is $17 \%$ the flow behaviour of the base changes and rheological parameters of cream base increases significantly (Table 6) due to change of its microstructure (Fig. 9).

In the absence of P338 a three-dimensional network is formed in the dispersion medium from the mixed aggregates of surfactants and CSA, due to which a coagulation structure is formed; this coagulation structure causes the rheological properties of the disperse system (Fig. 7, 8; Table 6) $[9,10]$. At a P338 concentration of $17 \%$, lyotropic liquid crystals containing a mixture of CPC and CSA are not formed due to the interaction of surfactants with P338; instead, the CSA as fine particles form the dispersed phase of the cream base. It can be assumed that CPC together with P338 are adsorbed on the surface of dispersed CSA particles, and P338, dissolved in the dispersion medium, «crosslinks» individual particles of the dispersed phase with adsorption layers by ion-dipole and steric interactions with CPC. With a sufficient content of $\mathrm{P} 338$, this three-dimensional network is formed through the entire volume of the dispersed system, which lead to a change in the flow behaviour (Fig. 7, 8). This phenomenon occurs in emulsions $\mathrm{o} / \mathrm{w}$ and in cream bases without the oil phase, as well as when cationic, anionic and nonionic surfactants are used as emulsifier $\mathrm{o} / \mathrm{w}$ (Fig. 7, 8). It should be noted that at temperatures above the melting point of $\mathrm{CSA}$, which can range from $49^{\circ} \mathrm{C}$ to $56{ }^{\circ} \mathrm{C}$ [1], cream bases without oil phase with the described microstructure are emulsions $\mathrm{o} / \mathrm{w}$, and at temperatures below the melting point of CSA they become suspensions.

In the case of the cream base without P338, the apparent viscosity of this base decrease with increasing temperature, at about $55^{\circ} \mathrm{C}$ the base melts and becomes a Newtonian fluid (Fig. 10, line 1). In the presence of $17 \%$ P338 in the base, a structured system with high apparent viscosity is formed at $70^{\circ} \mathrm{C}$; in the temperature range from $55^{\circ} \mathrm{C}$ to $25^{\circ} \mathrm{C}$, the apparent viscosity is approximately the same (Fig. 10, line 2). When this base is exposed to shear stress their apparent viscosity decreases due to thixotropic properties, but it is completely restored 
during holding without stirring in contrast to the base without P338 (Table 7).

P338 provided strong enough adhesion in the case of both solutions and cream bases (Table 8). Strength of adhesion of cream base with $17 \%$ P338 was 3.5 times greater than adhesion of cream base without P338. It should be noted that among objects under study, the cream base with $17 \%$ P338 had high apparent viscosity together with the strong enough adhesion (Tables 6,8). The combination of such properties is optimal for creams intended for vaginal use.

P338 binds water, as a result the value of $\tau_{-1}$ of the probe TEMPON increases linearly with increasing P338 content (Fig. 3, b). Due to high molecular mass (from 12,700 to $17,400[1,2])$ P338 molecules are not able to diffuse through the membrane but solutions of P338 absorb water diffusing to them through a membrane (Fig. 11, Table 9).

The $s o l \rightarrow g e l$ transition, which occurs in solutions when P338 content is increasing from $17 \%$ to $20 \%$, has little effect on the rate of water absorption and cumulative amount of absorbed water. On the contrary, the change in the microstructure of the cream base, caused by introduction of $17 \% \mathrm{P} 338$, leads to a significant decrease in water absorption parameters (Fig. 11, Table 9). The research results show that significant changes in the properties of this cream base (increase in rheological parameters and decrease in water absorption) are primarily due to the formation of a three-dimensional network due to the interaction of P338 with adsorption layers on CSA particles. According to the results of the study regarding water absorption, the prolonged release of active substances dissolved in the dispersion medium of the cream base might be expected. Therefore, further studies concerning the in vitro release of active substances from bases containing poloxamers should be considered promising.

Under the impact of $17 \%$ P338 modification of the properties of cream bases containing surfactants and CSR occurs. Such bases are characterized by high rheological parameters, strong adhesion, prolonged osmotic activity with slight ability to dehydrate of biological objects. Therefore, these bases are promising for development of semi-solid preparations for topical application on mucous membranes, skin and wounds (in the proliferative phase of the wound healing) [6].

This article presents a study regarding the interaction surfactants with P338, as well as the effect of P338 on the properties of cream bases. According to the obtained results, similar studies using different poloxamers 188, 237, $407[1,2]$ seem to be reasonable, which would clarify their peculiarities concerning their performance characteristics in the composition of semi-solid preparations. Furthermore, the studies presented here were limited to the use of certain excipients such as surfactants. According to the literature [21], for the pharmaceutical development of semi-solid preparations relevant studies on the effect of hydrophilic non-aqueous solvents on the structure of the poloxamer associates and the functional properties of these excipients might be advisable. For this purpose, research by rotational viscometry and spin probes method is promising. Probably, solutions with higher concentrations of poloxamers should also be studied within certain temperature ranges when various lyotropic liquid crystals are formed.

\section{Conclusions}

Under the effect of P338, the hydrodynamic diameter of the micelles of cationic, anionic, and nonionic surfactants decreases, the absolute value of their $\zeta$-potential decreases, and the microviscosity of micelle nuclei increases. The change in the structure of aggregates of surfactant with fatty alcohols also occurs; EPR spectra, which were superpositions characteristic of lateral phase separation, are converted into triplets which is evidence of uniform distribution of lipophilic probes in the phase of surfactant. That is, under impact of P338 the structure of surfactant micelles and aggregates of surfactant with fatty alcohols changes due to the interaction of surfactants and P338. As a result of this interaction at a sufficiently high P338 concentration (17\%), the rheological parameters of the bases increase significantly, their flow behaviour and microstructure change. The rheological parameters of the bases continue to be high in the temperature range from $25^{\circ} \mathrm{C}$ to $70^{\circ} \mathrm{C}$, and their apparent viscosity is completely restored after prolong exposure of shear stress. P338 enhances the adhesive properties of the bases. Due to their microstructure, cream bases have a lower ability to absorb water compared to a solution and gel containing $17 \%$ and $20 \%$ P338, respectively. Taking into account their properties, cream bases with sufficient content of P338 are promising for the development of medicinal products for topical application on mucous membranes, skin and wounds (in the proliferative phase of the wound healing).

\section{Conflict of interests}

The authors declare that they have no conflicts of interest.

\section{Financing}

The study was performed without financial support.

\section{References}

1. The European Pharmacopoeia (2019). European Directorate for the Quality of Medicines \& HealthCare of the Council of Europe. Strasbourg, 5224.

2. Sheskey, P. J., Hancock, B. C., Moss, G. P., Goldfarb, D. J. (Eds.) (2020). Handbook of Pharmaceutical Excipients,London: Pharm. Press, 1296.

3. Derzhavnyi reiestr likarskykh zasobiv Ukrainy. Available at: http://www.drlz.kiev.ua/

4. Gosudarstvenniy reestr lekarstvennykh sredstv (GRLS). Available at: http://grls.rosminzdrav.ru

5. Liapunov, N. A., Bezuglaia, E. P., Fadeikina, A. G., Lysokobylka, A. A., Stolper, Iu. M. (1999). Sozdanie miagkikh lekarstvennykh sredstv na razlichnykh osnovakh. Soobschenie 1. Issledovanie reologicheskikh svoistv mazei na vodorastvorimykh osnovakh. Farmakom, 6, 10-16. 
6. Datsenko, B. M. (Ed.) (1995). Teoryia y praktyka mestnoho lechenyia hnoinikh ran. Kyiv: Zdorovia, 384.

7. Da Silva, J. B., Cook, M. T., Bruschi, M. L. (2020). Thermoresponsive systems composed of poloxamer 407 and HPMC or NaCMC: mechanical, rheological and sol-gel transition analysis. Carbohydrate Polymers, 240. doi: http://doi.org/10.1016/ j.carbpol.2020.116268

8. De Souza Ferreira, S. B., Bruschi, M. L. (2021). Investigation of the physicochemical stability of emulgels composed of poloxamer 407 and different oil phases using the Quality by Design approach. Journal of Molecular Liquids, 332. doi: http://doi.org/ 10.1016/j.molliq.2021.115856

9. Lyapunova, A. M., Bezugla, O. P., Lyapunov, O. M. (2017). The study of o/w emulsions using the rotating viscometer method and the method of spin probes. News of Pharmacy, 4 (92), 29-34. doi: http://doi.org/10.24959/nphj.17.2190

10. Liapunov, M. O., Ivanov, L. V., Bezuhla, O. P., Zhdanov, R. I., Tsymbal, L. V. (1992). Doslidzhennia ahrehativ poverkhnevo-aktyvnykh rechovyn (PAR) metodom spinovykh zondiv. Farmatsevtychnyi zhurnal, 5-6, 40-45.

11. Bodratti, A., Alexandridis, P. (2018). Formulation of Poloxamers for Drug Delivery. Journal of Functional Biomaterials, 9 (1), 11. doi: http://doi.org/10.3390/jfb9010011

12. Fakhari, A., Corcoran, M., Schwarz, A. (2017). Thermogelling properties of purified poloxamer 407. Heliyon, 3 (8). doi: http://doi.org/10.1016/j.heliyon.2017.e00390

13. Russo, E., Villa, C. (2019). Poloxamer Hydrogels for Biomedical Applications. Pharmaceutics, 11 (12), 671. doi: http:// doi.org/10.3390/pharmaceutics11120671

14. Abdeltawab, H., Svirskis, D., Sharma, M. (2020). Formulation strategies to modulate drug release from poloxamer based in situ gelling systems. Expert Opinion on Drug Delivery, 17 (4), 495-509. doi: http://doi.org/10.1080/17425247.2020.1731469

15. Ci, L., Huang, Z., Liu, Y., Liu, Z., Wei, G., Lu, W. (2017). Amino-functionalized poloxamer 407 with both mucoadhesive and thermosensitive properties: preparation, characterization and application in a vaginal drug delivery system. Acta Pharmaceutica Sinica B, 7 (5), 593-602. doi: http://doi.org/10.1016/j.apsb.2017.03.002

16. Francke, N. M., Bunjes, H. (2021). Drug localization and its effect on the physical stability of poloxamer 188-stabilized colloidal lipid emulsions. International Journal of Pharmaceutics, 599. doi: http://doi.org/10.1016/j.ijpharm.2021.120394

17. Kumar, K., Dhawan, N., Sharma, H., Vaidya, S., Vaidya, B. (2013). Bioadhesive polymers: Novel tool for drug delivery. Artificial Cells, Nanomedicine, and Biotechnology, 42 (4), 274-283. doi: http://doi.org/10.3109/21691401.2013.815194

18. Giuliano, E., Paolino, D., Fresta, M., Cosco, D. (2018). Mucosal Applications of Poloxamer 407-Based Hydrogels: An Overview. Pharmaceutics, 10 (3), 159. doi: http://doi.org/10.3390/pharmaceutics10030159

19. Cook, M. T., Brown, M. B. (2018). Polymeric gels for intravaginal drug delivery. Journal of Controlled Release, 270 , 145-157. doi: http://doi.org/10.1016/j.jconrel.2017.12.004

20. Soliman, K. A., Ullah, K., Shah, A., Jones, D. S., Singh, T. R. R. (2019). Poloxamer-based in situ gelling thermoresponsive systems for ocular drug delivery applications. Drug Discovery Today, 24 (8), 1575-1586. doi: http://doi.org/10.1016/ j.drudis.2019.05.036

21. Ivanova, R., Alexandridis, P., Lindman, B. (2001). Interaction of poloxamer block copolymers with cosolvents and surfactants. Colloids and Surfaces A: Physicochemical and Engineering Aspects, 183-185, 41-53. doi: http://doi.org/10.1016/ s0927-7757(01)00538-6

22. Alexandridis, P., Alan Hatton, T. (1995). Poly(ethylene oxide)-poly(propylene oxide)-poly(ethylene oxide) block copolymer surfactants in aqueous solutions and at interfaces: thermodynamics, structure, dynamics, and modeling. Colloids and Surfaces A: Physicochemical and Engineering Aspects, 96 (1-2), 1-46. doi: http://doi.org/10.1016/0927-7757(94)03028-x

23. Ćirin, D., Krstonošić, V., Poša, M. (2017). Properties of poloxamer 407 and polysorbate mixed micelles: Influence of polysorbate hydrophobic chain. Journal of Industrial and Engineering Chemistry, 47, 194-201. doi: http://doi.org/10.1016/j.jiec.2016.11.032

24. Kuznetsov, A. N. (1976). Metod spinovogo zonda (Osnovy i primenenie). Moscow: Nauka, 210.

25. Likhtenshtein, G. I. (1974). Metod spinovykh zondov v molekuliarnoi biologii. Moscow: Nauka, 256.

26. Maslii, Y., Ruban, O., Levachkova, Y., Gureyeva, S., Kolisnyk, T. (2020). Choice of mucosal adhesive in the composition of a new dental gel. Pharmakeftiki, 32 (I), 40-49.

27. Derzhavna Farmakopeia Ukrainy. Vol. 1 (2015). Kharkiv: Derzhavne pidpryiemstvo «Ukrainskyi naukovyi farmakopeinyi tsentr yakosti likarskykh zasobiv», 1128.

28. Bezuglaya, E., Ivashchenko, H., Lyapunov, N., Zinchenko, I., Liapunova, A., Stolper, Y. et. al. (2021). Study of factors affecting the in vitro release of diclofenac sodium from hypromelose-based gels. ScienceRise: Pharmaceutical Science, 5 (33), $12-31$. doi: http://doi.org/10.15587/2519-4852.2021.243040

29. Rusanov, A. I., Schekin, A. K. (2016). Mitselloobrazovanie v rastvorakh poverkhnostno-aktivnykh veschestv. Saint Petersburg: OOO «Izdatelstvo «Lan», 612.

Received date 16.11.2021

Accepted date 17.12.2021

Published date 30.12.2021

Elena Bezuglaya*, PhD, Senior Researcher, Head of Laboratory, Laboratory of Technology and Analysis of Medicinal Products, State Scientific Institution "Institute for Single Crystals" of National Academy of Sciences of Ukraine, Nauky ave., 60, Kharkiv, Ukraine, 61072 
Nikolay Lyapunov, Doctor of Pharmaceutical Sciences, Professor, Leading Researcher, Laboratory of Technology and Analysis of Medicinal Products, State Scientific Institution "Institute for Single Crystals" of National Academy of Sciences of Ukraine, Nauky ave., 60, Kharkiv, Ukraine, 61072

Oleksii Lysokobylka, Junior Researcher, Laboratory of Technology and Analysis of Medicinal Products, State Scientific Institution “Institute for Single Crystals” of National Academy of Sciences of Ukraine, Nauky ave., 60, Kharkiv, Ukraine, 61072

Oleksii Liapunov, PhD, Researcher, Laboratory of Technology and Analysis of Medicinal Products, State Scientific Institution "Institute for Single Crystals" of National Academy of Sciences of Ukraine, Nauky ave., 60, Kharkiv, Ukraine, 61072

Volodimir Klochkov, PhD, Senior Researcher, Department of Nanostructured Materials, State Scientific Institution "Institute for Scintillation Materials" of National Academy of Sciences of Ukraine, Nauky ave., 60, Kharkiv, Ukraine, 61072

Hanna Grygorova, PhD, Senior Researcher, Department of Nanostructured Materials, State Scientific Institution "Institute for Scintillation Materials" of National Academy of Sciences of Ukraine, Nauky ave., 60, Kharkiv, Ukraine, 61072

Anna Liapunova, $\mathrm{PhD}$, Researcher, Laboratory of Technology and Analysis of Medicinal Products, State Scientific Institution "Institute for Single Crystals" of National Academy of Sciences of Ukraine, Nauky ave., 60, Kharkiv, Ukraine, 61072

*Corresponding author: Elena Bezuglaya, e-mail: bezugla.op@gmail.com 